\title{
A pseudo-compressible variational multiscale solver for turbulent incompressible flows
}

\author{
Liang Yang · Santiago Badia • Ramon \\ Codina
}

Received: date / Accepted: date

\begin{abstract}
In this work, we design an explicit time-stepping solver for the simulation of the incompressible turbulent flow through the combination of VMS methods and artificial compressibility. We evaluate the effect of the artificial compressibility on the accuracy of the explicit formulation for under-resolved LES simulations. A set of benchmarks have been solved, e.g., the 3D Taylor-Green vortex problem in turbulent regimes. The resulting method is proven to be an effective alternative to implicit methods in some application ranges (in terms of problem size and computational resources), providing comparable results with very low memory requirements. As an example, with the explicit approach, we are able to solve accurately the Taylor-Green vortex benchmark in a fine mesh with $512^{3}$ cells on a 12 cores 64 GB ram machine.
\end{abstract}

Keywords matrix-free $\cdot$ artificial compressibility method · variational multiscale method $(\mathrm{VMS}) \cdot$ explicit time stepping $\cdot$ turbulent incompressible flows

\section{Introduction}

The presence of turbulence, which is always produced at high Reynolds numbers, poses a challenge for the numerical simulation of the Navier-Stokes equations. Capturing all the scales of the turbulent flow, known as the "Direct Numerical Simulation" (DNS) [28], or even only the larger scales in "Large Eddy Simulation" (LES), involve large scale problems on very fine grids and small time steps

Liang Yang

School of Mathematics, Computer Science \& Engineering,

City University London, Northampton Square, London EC1V 0HB, UK

E-mail: liang.yang.1@city.ac.uk

Santiago Badia

Centre Internacional de Metodes Numerics en Enginyeri (CIMNE), Universitat Politècnica de Catalunya (UPC), 08860 Castelldefels, Spain

E-mail: sbadia@cimne.upc.edu

Roman Codina

Universitat Politècnica de Catalunya (UPC), Campus Nord, Jordi Girona 1-3, Edifici C1, 08034 Barcelona, Spain 
[31]. Taking into account the small-scale flow features in both time and space, explicit methods with small time steps is an appealing choice. In this sense, it is reasonable to envisage an increasing interest on matrix-free schemes as researchers will struggle with the draconian memory requirements of the forthcoming supercomputers.

Grid-based methods can be combined with explicit time integration when supplemented with an artificial compressibility method. Intensive research in this area has been done for laminar flows, within the framework of finite difference methods [9], finite volume methods [26], or the characteristic-based splitting methods [29]. In this work, we will develop explicit finite element (FE) schemes for turbulent flows. For FE schemes, the first problem when discretising the Navier-Stokes equations using FE methods is the fact that traditional Galerkin-based FE schemes are not suitable for convection dominated flows when dealing with under-resolved simulations. The second problem is the compatibility requirement between the velocity and pressure FE spaces for stability purposes, which is known as the LBB condition. The variational multiscale method (VMS) framework is introduced to overcome these difficulties [22]. The VMS approach can be understood as a residual-based stabilised FE method, and so, the singularly perturbed nature of the Navier-Stokes equations is stabilised as the Reynolds number increases. On the other hand, it allows one to avoid the compliance of the compatibility conditions (the so-called discrete inf-sup) between velocity and pressure spaces, and so, we are able to use equal-order interpolation.

The VMS concept is an LES turbulent model for the Navier-Stokes equations, as suggested in [10], exploited in [23], and analytically justified in [21]. One further development of the VMS framework was to consider the subscale proportional to the orthogonal component of the residual with respect to the FE spaces, leading to the orthogonal subscales VMS technique [14]. Another improvement was to consider a transient evolution equation for the subscale component, leading to the dynamic subscales in [13]. In these situations, the projection-based VMS method uses variational projections similar to the traditional filtered equations, which provides a numerically oriented turbulent modelling feature. Compared with classical LES-type filters, the VMS method does not have the problem associated to inhomogeneous non-commutative filters for wall-bounded flows. Further, the VMS method retains numerical consistency in the FE equations, whereas Smagorinskytype models introduce an error of order $h^{\frac{4}{3}}$. Good numerical results have been obtained with this approach, which demonstrate its capability to compete with traditional LES turbulence modelling approaches (see e.g. $[5,16]$ ).

The numerical solution of the VMS discretisation of incompressible flow is traditionally obtained using either a pressure segregation scheme [1] or monolithic approaches [5], which require to solve linear systems and (possibly) nonlinear iterations. For large scale problems, the linear system is solved via Krylov iterative solvers. In order to solve large scale problems with implicit methods, the main requirement is the use of weakly scalable preconditioning techniques [3, 4,30].

So far, VMS methods have been restricted to implicit time stepping techniques. However, explicit methods (or matrix-free lightweight implicit methods) are favoured in some situations, e.g., when one wants to tackle larger problems with limited computational resources. As a result, in this paper we investigate the possibility of imposing an explicit time-stepping approach for the simulation of the incompressible turbulent flow through the combination of VMS methods and 
artificial compressibility. We show that the resulting scheme, after the artificial compressibility perturbation, can still provide accurate under-resolved LES simulations of turbulent flows with a very limited memory consumption. The proposed scheme can be straightforwardly applied to structured and unstructured meshes. In particular, (multi-)linear equal-order velocity and pressure interpolation is used in this work. Compared to other explicit methods, the built-in sub-grid component acts as an implicit LES turbulence model and no extra turbulent modelling is required.

This paper is split into the following sections. In Sect. 2, the governing equations of the Navier-Stokes problem and its artificial compressibility formulation are presented. Sect. 3 combines the VMS formulation with the artificial compressibility technique. Sect. 4 presents a range of numerical results in order to demonstrate the aspects described in previous sections, including the 2-D cavity flow, 2-D plane mixing layer, 3-D cavity flow and 3-D Taylor Green vortex. Finally, some concluding remarks are made in Sect. 5.

\section{Governing equations}

\subsection{Incompressible Navier-Stokes equations}

Let $\Omega$ be a bounded domain of $\mathbb{R}^{d}$ where $d=2$ or 3 is the spatial dimension, with boundary $\Gamma=\partial \Omega$, and let the time interval be $[0, T]$. The strong form of the incompressible Navier-Stokes problem consists of finding the velocity field $\boldsymbol{u}$ and pressure $p$ such that

$$
\begin{aligned}
\partial_{t} \boldsymbol{u}-\nu \nabla^{2} \boldsymbol{u}+\boldsymbol{u} \cdot \boldsymbol{\nabla u}+\boldsymbol{\nabla} p=\boldsymbol{f} & \text { in } \Omega \times(0, T), \\
\boldsymbol{\nabla} \cdot \boldsymbol{u}=0 & \text { in } \Omega \times(0, T),
\end{aligned}
$$

where $\boldsymbol{f}$ is the force vector and $\nu>0$ is the kinematic viscosity. System (1) has to be supplied with appropriate boundary and initial conditions. The boundary $\Gamma$ is divided into the Dirchilet boundary $\Gamma_{D}$ and Neumann boundary $\Gamma_{N}$, satisfy $\Gamma_{D} \cup \Gamma_{N}=\Gamma$ and $\Gamma_{D} \cap \Gamma_{N}=\varnothing$. Then, the boundary and initial conditions read:

$$
\begin{aligned}
& \boldsymbol{u}=\overline{\boldsymbol{u}} \quad \text { on } \Gamma_{D} \times(0, T), \\
& \left(-p \boldsymbol{I}+\nu\left(\nabla u+(\boldsymbol{\nabla} u)^{T}\right)\right) \cdot \boldsymbol{n}=\overline{\boldsymbol{t}} \quad \text { on } \Gamma_{N} \times(0, T), \\
& \boldsymbol{u}=\boldsymbol{u}_{0} \quad \text { in } \Omega \times\{0\},
\end{aligned}
$$

where $\boldsymbol{n}$ is the unit outward vector normal to the surface $\Gamma$. To simplify the exposition, we consider the pure Dirichlet boundary $\Gamma_{D} \equiv \Gamma$.

\subsection{Artificial compressibility method}

The artificial compressibility formulation replaces the divergence free constraint by adding a pseudo-time derivative of the pressure. System (1) is modified to

$$
\begin{aligned}
\partial_{t} \boldsymbol{u}-\nu \boldsymbol{\nabla}^{2} \boldsymbol{u}+\boldsymbol{u} \cdot \boldsymbol{\nabla} \boldsymbol{u}+\boldsymbol{\nabla} p=\boldsymbol{f} & \text { in } \Omega \times(0, T) \\
\partial_{t} p+\varepsilon^{-1} \boldsymbol{\nabla} \cdot \boldsymbol{u}=0 & \text { in } \Omega \times(0, T)
\end{aligned}
$$


In $(3), \varepsilon^{-1}$ is the artificial compressibility coefficient or pseudo compressibility coefficient, where an artificial sound speed can be evaluated as $c=\sqrt{\varepsilon^{-1}}$. As the pressure wave speed $c$ reaches a very large number, (3) approximates (1). A relaxation of the incompressibility will allow one to use an explicit time stepping scheme. However, a large artificial pressure wave speed will be a limit factor of the explicit time stepping. The characteristic velocity $\varepsilon^{-1}=c^{2}=\left(\operatorname{ASS}\|\boldsymbol{u}\|_{\infty}\right)^{2}$, where $\|\boldsymbol{u}\|_{\infty}$ is the maximum norm of the velocity field, and ASS is the artificial sound speed coefficient usually takes a value from 1 and 10. For the steady state problem, the coefficient will not effect the final solution because the residual will convergence to 0 . The effect of ASS for turbulent flows will be investigated in the transient numerical example, 2D plane mixing layer and 3D Taylor Green vortex. The numerical experiments indicate that values of ASS 5 provide a good enough satisfaction of the incompressibility constraint.

Notice that the pressure behaviour in (3) changes from elliptic to hyperbolic (wave-propagation) character with the artificial compressibility method, and the Courant-Friedrichs-Lewy (CFL) condition is a necessary condition for temporal stability. In particular, for the problem considered the time step size $\Delta t$ has to be such that

$$
\Delta t \leq \alpha \frac{\Delta h}{c},
$$

where $\alpha$ is a factor that depends on the time integration scheme being used and the safety margin desired, and $\Delta h$ is the minimum mesh size. In what follows, we will assume that the time discretisation is uniform, with time step size $\Delta t$. Time step levels will be denoted with a superscript.

\subsection{Weak formulation and notation}

Let us introduce some notation. $L^{2}(\Omega)$ denotes the spaces of functions that are square integrable in $\Omega$

$$
L^{2}(\Omega) \doteq\left\{u: \Omega \rightarrow \mathbb{R} \mid \int_{\Omega} u^{2} d \Omega<\infty\right\}
$$

It is a Hilbert space with scalar product

$$
(u, v)_{\Omega} \equiv(u, v) \doteq \int_{\Omega} u(\boldsymbol{x}) v(\boldsymbol{x}) d \Omega .
$$

When the integral is performed over a subdomain $\omega$, we will denote it by $(\cdot, \cdot)_{\omega}$. $H^{1}(\Omega)$ denotes the Sobolev space of square integrable functions with square integral derivative as

$$
H^{1}(\Omega) \doteq\left\{u: \Omega \rightarrow \mathbb{R} \mid u, \nabla u \in L^{2}(\Omega)\right\} .
$$

We define the solution $\boldsymbol{H}_{E}^{1}(\Omega)$ space and the test space $\boldsymbol{H}_{0}^{1}(\Omega)$ by

$$
\begin{aligned}
& \boldsymbol{H}_{E}^{1}(\Omega) \doteq\left\{\boldsymbol{u} \in H^{1}(\Omega)^{d} \mid \boldsymbol{u}=\overline{\boldsymbol{u}} \text { on } \Gamma\right\}, \\
& \boldsymbol{H}_{0}^{1}(\Omega) \doteq\left\{\boldsymbol{u} \in H^{1}(\Omega)^{d} \mid \boldsymbol{u}=\mathbf{0} \text { on } \Gamma\right\},
\end{aligned}
$$


respectively. Moreover, $\boldsymbol{H}^{-1}(\Omega)$ denote the topological dual of $\boldsymbol{H}_{0}^{1}(\Omega)$ and $\langle\cdot, \cdot\rangle$ the duality pairing between $\boldsymbol{H}^{-1}(\Omega)$ and $\boldsymbol{H}_{0}^{1}(\Omega)$. Then, the standard weak form of (3) is the following: for each time $t$, find a velocity $\boldsymbol{u}(\cdot, t) \in \boldsymbol{H}_{E}^{1}(\Omega)$ and a pressure $p(\cdot, t) \in L^{2}(\Omega)$ such that

$$
\begin{aligned}
\left(\partial_{t} \boldsymbol{u}, \boldsymbol{v}\right)+\nu(\boldsymbol{\nabla} \boldsymbol{u}, \boldsymbol{\nabla v})-(p, \boldsymbol{\nabla} \cdot \boldsymbol{v})+\langle\boldsymbol{u} \cdot \boldsymbol{\nabla} \boldsymbol{u}, \boldsymbol{v}\rangle & =\langle\boldsymbol{f}, \boldsymbol{v}\rangle & & \text { for all } \boldsymbol{v} \in \boldsymbol{H}_{0}^{1}(\Omega), \\
\left(\partial_{t} p, q\right)+\varepsilon^{-1}(\boldsymbol{\nabla} \cdot \boldsymbol{u}, q) & =0 & & \text { for all } q \in L^{2}(\Omega) .
\end{aligned}
$$

\subsection{Galerkin FE method}

Given a FE partition of the domain $\Omega$, the problem is to construct a velocity solution space $\boldsymbol{V}_{h}^{E} \subset \boldsymbol{H}_{E}^{1}(\Omega)$ and $L_{h} \subset L^{2}(\Omega)$ to set the discrete problem: for each time $t$, find a velocity $\boldsymbol{u}_{h}(\cdot, t) \in \boldsymbol{V}_{h}^{E}$ and a pressure $p(\cdot, t)_{h} \in L_{h}$ such that

$$
\begin{aligned}
\left(\partial_{t} \boldsymbol{u}_{h}, \boldsymbol{v}_{h}\right)+\nu\left(\boldsymbol{\nabla} \boldsymbol{u}_{h}, \boldsymbol{\nabla} \boldsymbol{v}_{h}\right)-\left(p_{h}, \boldsymbol{\nabla} \cdot \boldsymbol{v}_{h}\right)+\left\langle\boldsymbol{u}_{h} \cdot \boldsymbol{\nabla} \boldsymbol{u}_{h}, \boldsymbol{v}_{h}\right\rangle & =\left\langle\boldsymbol{f}, \boldsymbol{v}_{h}\right\rangle \\
\left(\partial_{t} p_{h}, q_{h}\right)+\varepsilon^{-1}\left(\boldsymbol{\nabla} \cdot \boldsymbol{u}_{h}, q_{h}\right) & =0
\end{aligned}
$$

for all $\boldsymbol{v}_{h} \in \boldsymbol{V}_{h}^{0}$ and $q_{h} \in L_{h}$, where $\boldsymbol{V}_{h}^{0}$ is constructed as $\boldsymbol{V}_{h}^{E}$ but with homogeneous boundary conditions. As commented above, it is well known that (6) suffers from numerical instabilities for high Reynolds number problems. Also, the velocity and pressure solution space must satisfy the inf-sup condition in order to have a well-posed problem, with bounded pressure field. These difficulties can be handled by using the VMS method.

\section{Explicit Variational multiscale method}

In general, the VMS method decomposes the solution $\boldsymbol{u}$ and $p$ on a large scale component and a subscale component. The large scale components $\boldsymbol{u}_{h}$ and $p_{h}$ are resolved by the FE mesh, whilst the subscale components $\tilde{\boldsymbol{u}}$ and $\tilde{p}$ are approximated by a certain analytical approach. Let us consider a scale decomposition of spaces $\boldsymbol{H}_{E}^{1}(\Omega)$ and $L^{2}(\Omega)$ such that $\boldsymbol{H}_{E}^{1}(\Omega)=\boldsymbol{V}_{h} \oplus \tilde{\boldsymbol{V}}$ and $L^{2}(\Omega)=L_{h} \oplus \tilde{L}$, where $\tilde{\boldsymbol{V}}$ and $\tilde{L}$ denote the infinite-dimensional spaces that complete the FE spaces $\boldsymbol{V}_{h}$ and $L_{h}$ to approximate the velocity and pressure in the standard Galerkin FE method. $(\cdot)_{h}$ and $(\tilde{\cdot})$ denote the FE component and the sub-grid component, respectively. Thus, we approximate the velocity $\boldsymbol{u}$ and pressure $p$ by

$$
\boldsymbol{u} \approx \boldsymbol{u}_{h}+\tilde{\boldsymbol{u}}, \quad p \approx p_{h}+\tilde{p}
$$

where $\boldsymbol{u}_{h} \in \boldsymbol{V}_{h}, \tilde{\boldsymbol{u}} \in \tilde{\boldsymbol{V}}, p_{h} \in L_{h}, \tilde{p} \in \tilde{L}$ for each time $t$. Using this splitting in (5), yields

$$
\begin{aligned}
\left(\partial_{t}\left(\boldsymbol{u}_{h}+\tilde{\boldsymbol{u}}\right), \boldsymbol{v}\right)+\nu(\nabla & \left.\left(\boldsymbol{u}_{h}+\tilde{\boldsymbol{u}}\right), \boldsymbol{\nabla} \boldsymbol{v}\right)-\left(p_{h}+\tilde{p}, \boldsymbol{\nabla} \cdot \boldsymbol{v}\right) \\
+\left\langle\left(\boldsymbol{u}_{h}+\tilde{\boldsymbol{u}}_{h}\right) \cdot \boldsymbol{\nabla}\left(\boldsymbol{u}_{h}+\tilde{\boldsymbol{u}}_{h}\right), \boldsymbol{v}\right\rangle & =\langle\boldsymbol{f}, \boldsymbol{v}\rangle, \\
\left(\partial_{t}\left(p_{h}+\tilde{p}\right), q\right)+\varepsilon^{-1}\left(\boldsymbol{\nabla} \cdot\left(\boldsymbol{u}_{h}+\tilde{\boldsymbol{u}}_{h}\right), q\right) & =0 .
\end{aligned}
$$


3.1 FE scale problem

First we consider FE test function, i.e. $\boldsymbol{v}=\boldsymbol{v}_{h} \in \boldsymbol{V}_{h}^{0}$ and $q=q_{h} \in L_{h}$. The resolved large-scale component of (7) yields

$$
\begin{aligned}
\left(\partial_{t}\left(\boldsymbol{u}_{h}+\tilde{\boldsymbol{u}}\right), \boldsymbol{v}_{h}\right)+\nu\left(\boldsymbol{\nabla}\left(\boldsymbol{u}_{h}+\tilde{\boldsymbol{u}}\right), \boldsymbol{\nabla} \boldsymbol{v}_{h}\right)-\left(p_{h}+\tilde{p}, \boldsymbol{\nabla} \cdot \boldsymbol{v}_{h}\right) & \\
+\left\langle\left(\boldsymbol{u}_{h}+\tilde{\boldsymbol{u}}\right) \cdot \boldsymbol{\nabla}\left(\boldsymbol{u}_{h}+\tilde{\boldsymbol{u}}\right), \boldsymbol{v}_{h}\right\rangle & =\left\langle\boldsymbol{f}, \boldsymbol{v}_{h}\right\rangle, \\
\left(\partial_{t}\left(p_{h}+\tilde{p}\right), q_{h}\right)+\varepsilon^{-1}\left(\boldsymbol{\nabla} \cdot\left(\boldsymbol{u}_{h}+\tilde{\boldsymbol{u}}\right), q_{h}\right) & =0 .
\end{aligned}
$$

Let us elaborate on some of the terms of these equations:

- A very important ingredient of our approach is that we choose the sub-grid spaces to be orthogonal in the $L^{2}(\Omega)$-sense to the FE spaces. Therefore, the time derivative terms $\left(\partial_{t} \tilde{\boldsymbol{u}}, \boldsymbol{v}_{h}\right)$ and $\left(\partial_{t} \tilde{p}, q_{h}\right)$ vanish:

$$
\left(\partial_{t}\left(\boldsymbol{u}_{h}+\tilde{\boldsymbol{u}}\right), \boldsymbol{v}_{h}\right)=\left(\partial_{t} \boldsymbol{u}_{h}, \boldsymbol{v}_{h}\right) ; \quad\left(\partial_{t}\left(p_{h}+\tilde{p}\right), q_{h}\right)=\left(\partial_{t} p_{h}, q_{h}\right)
$$

We show below how to satisfy this orthogonality in practice.

- The viscous term $\nu\left(\boldsymbol{\nabla}\left(\boldsymbol{u}_{h}+\tilde{\boldsymbol{u}}\right), \boldsymbol{\nabla} \boldsymbol{v}_{h}\right)$ is simplified as

$$
\begin{aligned}
& \nu\left(\boldsymbol{\nabla}\left(\boldsymbol{u}_{h}+\tilde{\boldsymbol{u}}\right), \boldsymbol{\nabla} \boldsymbol{v}_{h}\right) \\
& =\nu\left(\boldsymbol{\nabla} \boldsymbol{u}_{h}, \boldsymbol{\nabla} \boldsymbol{v}_{h}\right)+\nu \sum_{K}\left[\left(-\tilde{\boldsymbol{u}}, \Delta \boldsymbol{v}_{h}\right)_{K}+\left(\tilde{\boldsymbol{u}}, \boldsymbol{n} \cdot \boldsymbol{\nabla} \boldsymbol{v}_{h}\right)_{\partial K}\right] \\
& =\nu\left(\boldsymbol{\nabla} \boldsymbol{u}_{h}, \boldsymbol{\nabla} \boldsymbol{v}_{h}\right) .
\end{aligned}
$$

The term $\left(-\tilde{\boldsymbol{u}}, \Delta \boldsymbol{v}_{h}\right)_{K}$ vanishes for linear elements. The term $\left(\tilde{\boldsymbol{u}}, \boldsymbol{n} \cdot \boldsymbol{\nabla} \boldsymbol{v}_{h}\right)_{\partial K}$ is usually neglected in practice, although it could be taken into account using the strategy proposed in [12].

- In order to avoid derivatives of the sub-grid component, the convective component can be re-written as

$$
\begin{aligned}
& \left\langle\left(\boldsymbol{u}_{h}+\tilde{\boldsymbol{u}}\right) \cdot \nabla\left(\boldsymbol{u}_{h}+\tilde{\boldsymbol{u}}\right), \boldsymbol{v}_{h}\right\rangle \\
& =\left\langle\left(\boldsymbol{u}_{h}+\tilde{\boldsymbol{u}}\right) \cdot \boldsymbol{\nabla} \boldsymbol{u}_{h}, \boldsymbol{v}_{h}\right\rangle-\left\langle\tilde{\boldsymbol{u}},\left(\boldsymbol{u}_{h}+\tilde{\boldsymbol{u}}\right) \cdot \nabla \boldsymbol{v}_{h}\right\rangle,
\end{aligned}
$$

where we have used the fact that $\boldsymbol{u}_{h}+\tilde{\boldsymbol{u}}$ is divergence free when integrating by parts.

- Again to avoid derivatives of the sub-grid scales, the mass conservation equation is re-written as

$\varepsilon^{-1}\left(\nabla \cdot\left(\boldsymbol{u}_{h}+\tilde{\boldsymbol{u}}\right), q_{h}\right)=\varepsilon^{-1}\left(\boldsymbol{\nabla} \cdot \boldsymbol{u}_{h}, q_{h}\right)-\varepsilon^{-1}\left(\tilde{\boldsymbol{u}}, \boldsymbol{\nabla} q_{h}\right)+\varepsilon^{-1}\left(\boldsymbol{n} \cdot \tilde{\boldsymbol{u}}, q_{h}\right)_{\partial \Omega}$,

after integration by parts. As before, the sub-grid scale boundary term is neglected.

Taking into account the previous considerations, the equations we have are:

$$
\begin{aligned}
& \left(\partial_{t} \boldsymbol{u}_{h}, \boldsymbol{v}_{h}\right)+\left\langle\boldsymbol{a} \cdot \boldsymbol{\nabla} \boldsymbol{u}_{h}, \boldsymbol{v}_{h}\right\rangle+\nu\left(\boldsymbol{\nabla} \boldsymbol{u}_{h}, \boldsymbol{\nabla} \boldsymbol{v}_{h}\right) \\
& -\left(p_{h}, \boldsymbol{\nabla} \cdot \boldsymbol{v}_{h}\right)-\left(\tilde{p}, \boldsymbol{\nabla} \cdot \boldsymbol{v}_{h}\right)-\left\langle\tilde{\boldsymbol{u}}, \boldsymbol{a} \cdot \boldsymbol{\nabla} \boldsymbol{v}_{h}\right\rangle=0, \\
& \left(\partial_{t} p_{h}, q_{h}\right)+\varepsilon^{-1}\left(\boldsymbol{\nabla} \cdot \boldsymbol{u}_{h}, q_{h}\right)-\varepsilon^{-1}\left(\tilde{\boldsymbol{u}}, \boldsymbol{\nabla} q_{h}\right)=0,
\end{aligned}
$$

where $\boldsymbol{a} \doteq \boldsymbol{u}_{h}+\tilde{\boldsymbol{u}}$. The problem will be closed once an approximation for the sub-grid scale velocity and pressure is proposed. 


\subsection{Local sub-grid scale problem}

In this section, we discuss the sub-grid scale formulation and then give the analytical approximation of the solution. As a result, the orthogonal sub-grid scale in an explicit VMS method will be given. The sub-grid scale equation reads (in strong form):

$$
\begin{array}{r}
\partial_{t} \tilde{\boldsymbol{u}}+\left(\boldsymbol{u}_{h}+\tilde{\boldsymbol{u}}\right) \cdot \boldsymbol{\nabla} \tilde{\boldsymbol{u}}-\nu \Delta \tilde{\boldsymbol{u}}+\nabla \tilde{p}=\boldsymbol{R}_{u}, \\
\partial_{t} \tilde{p}+\varepsilon^{-1} \boldsymbol{\nabla} \cdot \tilde{\boldsymbol{u}}=R_{p},
\end{array}
$$

where $\boldsymbol{R}_{u}$ and $R_{p}$ are appropriate residuals of the FE components defined as

$$
\begin{aligned}
\boldsymbol{R}_{u} & =-\mathcal{P}_{u}\left(\partial_{t} \boldsymbol{u}_{h}+\left(\boldsymbol{u}_{h}+\tilde{\boldsymbol{u}}\right) \cdot \boldsymbol{\nabla} \boldsymbol{u}_{h}-\nu \Delta \boldsymbol{u}_{h}+\nabla p_{h}-\boldsymbol{f}\right), \\
R_{p} & =-\mathcal{P}_{p}\left(\partial_{t} p_{h}+\varepsilon^{-1} \boldsymbol{\nabla} \cdot \boldsymbol{u}_{h}\right) .
\end{aligned}
$$

$\mathcal{P}_{u}$ and $\mathcal{P}_{p}$ are projection operators onto the sub-grid scale spaces of velocities and pressure. In the case in which we take the identity operator in both cases, we recover the original VMS method proposed [22]. Codina [11] proposed to enforce the subscales to be orthogonal to the FE velocity and pressure spaces. It leads to $\mathcal{P}_{u} \doteq \Pi_{u}^{\perp} \doteq 1-\Pi_{u}$, where $\Pi_{u}$ is the $L^{2}$-projector onto $\boldsymbol{V}_{h}$ (using mass lumping), i.e., for a function $f$, we compute the nodal values of $\Pi_{u}(f)$ at any node $a$ of the FE mesh as $\Pi_{u}(f)^{a}=\frac{\int_{\Omega} f \varphi^{a}}{\int \Omega \varphi^{a}}$, where $\varphi^{a}$ is the corresponding nodal shape function. We proceed analogously for $\mathcal{P}_{p}$ and $L_{h}$.

Next, we consider the following algebraic approximation of this nonlinear differential system. We refer to [10] for a detailed exposition of the sub-grid problem approximation, e.g., using a motivation based on Fourier transforms. After time discretisation, e.g., using an implicit-explicit first order time integration, the approximated dynamic sub-grid model for the momentum equation reads as: compute $\tilde{\boldsymbol{u}}^{n+1}$ from

$\frac{1}{\Delta t} \tilde{\boldsymbol{u}}^{n+1}+\frac{1}{\tau_{u}^{n}} \tilde{\boldsymbol{u}}^{n+1}=\frac{1}{\Delta t} \tilde{\boldsymbol{u}}^{n}+\boldsymbol{R}_{u}^{n}, \quad$ where $\quad \tau_{u}^{n}=\left(\frac{c_{1} \nu}{h^{2}}+\frac{c_{2}\left|\boldsymbol{u}_{h}^{n}+\tilde{\boldsymbol{u}}^{n}\right|}{h}\right)^{-1}$,

or analogously,

$$
\tilde{\boldsymbol{u}}^{n+1}=\frac{\tau_{t}^{n}}{\Delta t} \tilde{\boldsymbol{u}}^{n}+\tau_{t}^{n} \boldsymbol{R}_{u}^{n}, \quad \text { where } \quad \frac{1}{\tau_{t}^{n}}=\frac{1}{\Delta t}+\frac{1}{\tau_{u}}
$$

$c_{1}$ and $c_{2}$ being algorithmic constants, that we set to $c_{1}=4, c_{2}=2$ in the numerical experiments. We can treat the sub-grid scale term implicitly because it does not imply an increase in CPU cost. Note that the previous expressions for the sub-grid scales are required at the integration points.

The pressure sub-grid scale component can be treated in a similar way. Again, following the motivation explained in [10], this component can be found from:

$$
\frac{1}{\Delta t} \tilde{p}^{n+1}+\varepsilon^{-1} \frac{1}{\tau_{p}^{n}} \tilde{p}^{n+1}=\frac{1}{\Delta t} \tilde{p}^{n}+R_{p}^{n}, \quad \text { where } \quad \tau_{p}^{n}=\frac{h^{2}}{\tau_{u}^{n}} .
$$

This term is not really required to get stability and convergence bounds [2]. Further, the exhaustive numerical analysis in [16] advocates to switch off the pressure sub-grid component for turbulent flow simulations. In this work, we follow this approach. 
3.3 Time integration and final algorithm

The motivation for using an artificial compressibility method is the possibility to use an explicit time integration scheme of the flow equations. Any choice is open, but in this paper we have used a simple second order time integration scheme for the FE solution and a first order time integration for the velocity sub-grid scales, as described above. This is consistent since the sub-grid scales are multiplied by the stabilisation parameters, which are of the order of the time step size. Note that they essentially behave as the critical time step that would be found in an forward Euler time integration [15]. The solver has been implemented in our inhouse code using Fortran90 and parallelised using OpenMP. A 2D matrix-vector implementation is illustrated in Appendix. The final algorithm is depicted below. 
Algorithm: A pseudo-compressible VMS method for incompressible flows

- Read $\boldsymbol{u}_{h}^{0}, p_{h}^{0}$ and set $\tilde{\boldsymbol{u}}=\mathbf{0}$, loop over time step $n$

- Loop over elements, loop over integration points

1. Evaluate velocity prediction $\hat{\boldsymbol{u}}_{h}^{n+1}$ and pressure prediction $\hat{p}_{h}^{n+1}$ at time step $n+1$ using the second order approximation:

$$
\hat{\boldsymbol{u}}_{h}^{n+1}=\frac{3}{2} \boldsymbol{u}_{h}^{n}-\frac{1}{2} \boldsymbol{u}_{h}^{n-1}, \quad \hat{p}_{h}^{n+1}=\frac{3}{2} p_{h}^{n}-\frac{1}{2} p_{h}^{n-1} .
$$

2. Add the nonlinear sub-grid scale component for the convective velocity $a$ :

$$
\boldsymbol{a}=\hat{\boldsymbol{u}}_{h}^{n+1}+\tilde{\boldsymbol{u}}^{n} .
$$

3. Calculate the stabilisation parameters $\tau_{t}^{n}$ as in (8).

4. Evaluate velocity sub-grid scales:

$$
\tilde{\boldsymbol{u}}^{n+1}=\frac{\tau_{t}}{\Delta t} \tilde{\boldsymbol{u}}^{n}-\Pi_{u}^{\perp}\left[\tau_{t}\left(\boldsymbol{a} \cdot \boldsymbol{\nabla} \boldsymbol{u}_{h}^{n}+\boldsymbol{\nabla} p_{h}^{n}\right)\right]
$$

5. Update the velocity and pressure for the next time step $n+1$ by solving:

$$
\left(\boldsymbol{u}_{h}^{n+1}, \boldsymbol{v}_{h}\right)=\left(\boldsymbol{u}_{h}^{n}, \boldsymbol{v}_{h}\right)-\Delta t \mathcal{R}_{u}^{n}, \quad\left(q_{h}, p_{h}^{n+1}\right)=\left(q_{h}, p_{h}^{n}\right)-\Delta t \mathcal{R}_{p}^{n}
$$

for all test functions $\boldsymbol{v}_{h}$ and $q_{h}$, where

$$
\begin{aligned}
\boldsymbol{R}_{u}^{n}= & \left(\boldsymbol{a} \cdot \boldsymbol{\nabla} \hat{\boldsymbol{u}}_{h}^{n+1}, \boldsymbol{v}_{h}\right)+\nu\left(\boldsymbol{\nabla} \hat{\boldsymbol{u}}_{h}^{n+1}, \boldsymbol{\nabla} \boldsymbol{v}_{h}\right)-\left(\hat{p}_{h}^{n+1}, \boldsymbol{\nabla} \cdot \boldsymbol{v}_{h}\right) \\
& -\left(\tilde{\boldsymbol{u}}^{n}, \boldsymbol{a} \cdot \boldsymbol{\nabla} \boldsymbol{v}_{h}\right)-\left\langle\boldsymbol{f}, \boldsymbol{v}_{h}\right\rangle \\
\mathcal{R}_{p}^{n}= & \varepsilon^{-1}\left(\boldsymbol{\nabla} \cdot \hat{\boldsymbol{u}}_{h}^{n+1}, q_{h}\right)-\varepsilon^{-1}\left(\tilde{\boldsymbol{u}}^{n}, \boldsymbol{\nabla} q_{h}\right)
\end{aligned}
$$

- Prescribe boundary conditions

$$
\boldsymbol{u}_{h}^{n+1}=\overline{\boldsymbol{u}} \text { on } \partial \Omega
$$

\section{Numerical Examples}

In this section, we consider first two $2 \mathrm{D}$ problems to evaluate the explicit VMS method. Next, we apply the framework to two 3D turbulent flow benchmarks.

\subsection{Two-dimensional flow: lid-driven cavity}

The first problem considered here is the well documented benchmark case of the lid-driven cavity flow to test the proposed method and the implementation in 2D. A scheme of the test problem configuration is shown in Fig. 1 (a) with the top wall moving to the right at a velocity $u_{x}=1$. The bottom and two vertical walls are non-slip boundaries. 


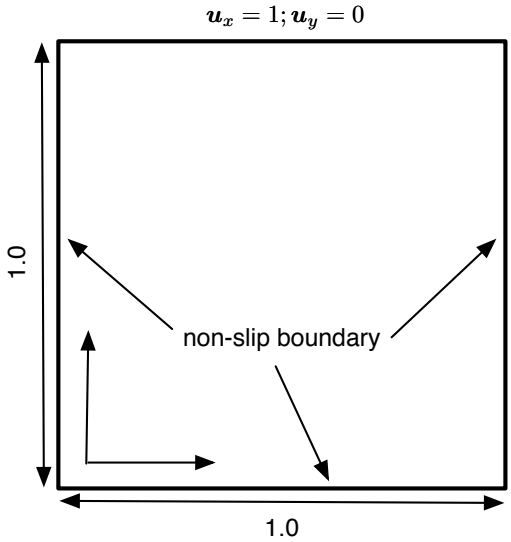

(a) Schematic diagram for the lid-driven (b) flow problem

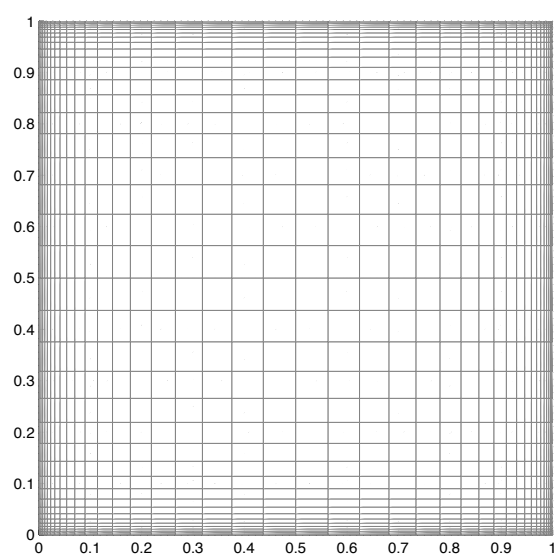

(b) Computational domain: a non-uniform structured meshes

Fig. 1 Two dimension lid driven flow

A $100 \times 100$ non-uniform structured mesh for $R e=100, R e=1000, R e=5000$ and $R e=10000$, containing finer grid points near the walls, is employed for the computation. Since the target is to compute the steady state, we do not study the effect of ASS in this case. The artificial sound speed is chosen to be $c=2\|\boldsymbol{u}\|_{\infty}^{2}$. The mesh grid point distance in the $x$ and $y$ directions is set to be a hyperbolic tangent profile. For example, the $y$ coordinates in terms of the $x$ coordinates are given by:

$$
y(x)=0.5+\frac{\tanh (5(x-0.5))}{2 \tanh (2.5)}, \quad 0 \leq x \leq 1,
$$

as shown in Fig. 1 (b).

The plots of vertical velocity profiles at the horizontal centreline and of horizontal velocity profiles at the vertical centreline, at different Reynolds numbers, are compared with the second order accurate solution by Ghia et at. [19] in Fig. 2. A good agreement with the reference result is observed.

We also present here the numerical results at $R e=100000$ performed on the uniform grids of $512^{2}, 1024^{2}$ and $2048^{2}$ for the transient case. The artificial sound speed is chosen to be $c=3\|\boldsymbol{u}\|_{\infty}^{2}$. In Fig. 3, it can be noticed that the coarse grid is more diffusive than the fine grid and the bilinear element under meshsize $1024^{2}$ is not fine enough to have a converged result for the lid-driven cavity flow under $R e=100000$. But the proposed method can capture the transient features of the cavity flow for very high Reynolds number. 


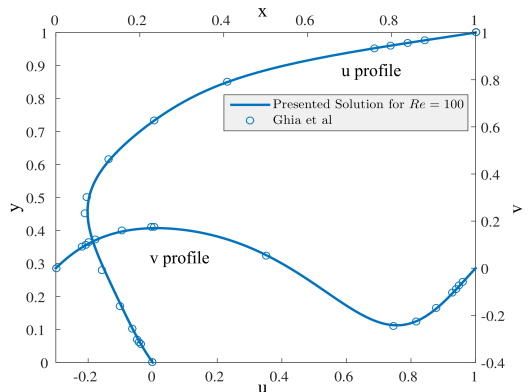

(a)

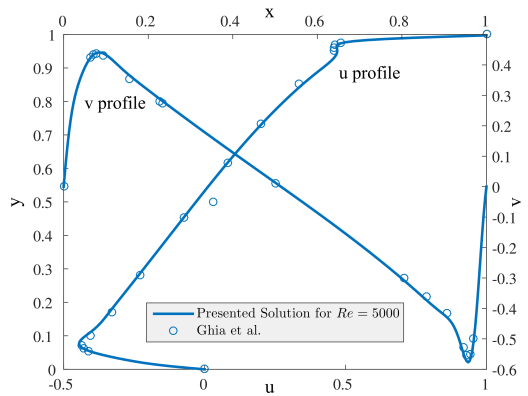

(c)

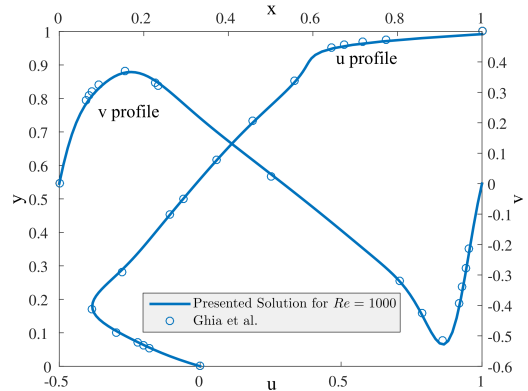

(b)

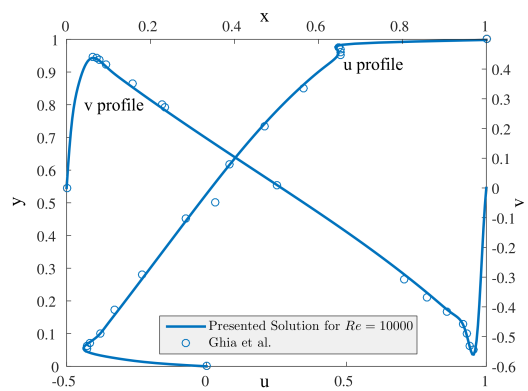

(d)

Fig. 2 2-D lid-driven flow. Comparison of velocity profiles with Ghia et at. [19] at the midsections for Reynolds number: (a) $R e=100$; (b) $R e=1000$; (c) $R e=5000$; (d) $R e=10000$.

\subsection{Two-dimensional flow: Kelvin-Helmholtz instability}

Kelvin-Helmholtz instability of a plane mixing layer is an important case in fluid dynamics. This example will not lead to what is usually perceived as turbulence, but it shows some features of the flow in common with turbulence in a certain sense, because the flow is extremely sensitive to the initial condition and a broadband energy spectrum of slope between $k^{-4}$ and $k^{-3}$ is developed [20]. The problem is set in the domain $\Omega=[0,1] \times[0,1]$. Free slip boundary conditions are applied at $y=0$ and $y=1$. At the left and right walls $x=0$ and $x=1$, periodic boundaries are imposed, as seen in Fig. 4.

According to $[6,20]$, the initial velocity field is set to be a hyperbolic tangent profile as:

$$
\begin{aligned}
& u_{x}=\|\boldsymbol{u}\|_{\infty}\left[\tanh \left(\frac{2 y-1}{\delta_{0}}\right)+c \frac{\partial \psi_{\text {per }}}{\partial y}\right] \\
& u_{y}=-\|\boldsymbol{u}\|_{\infty} c \frac{\partial \psi_{\text {per }}}{\partial x}
\end{aligned}
$$

where $u_{x}$ and $u_{y}$ are the velocity components, $\psi_{\text {per }}=\|\boldsymbol{u}\|_{\infty} \exp \left(-\frac{y-0.5}{\delta_{0}}\right)^{2} \cos (\alpha x)$ is a white noise perturbation expressed in a stream function formulation. The initial thickness is chosen as $\delta_{0}=1 / 28$ and the wave number as $\alpha=8 \pi$ to reach the 


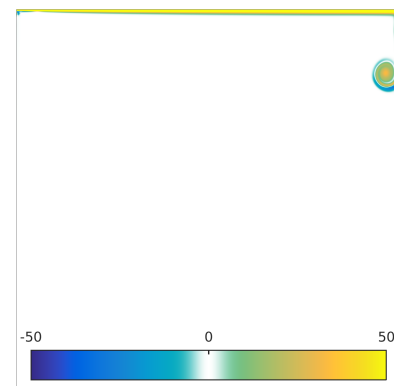

(a) $t=2$; grid: $512^{2}$

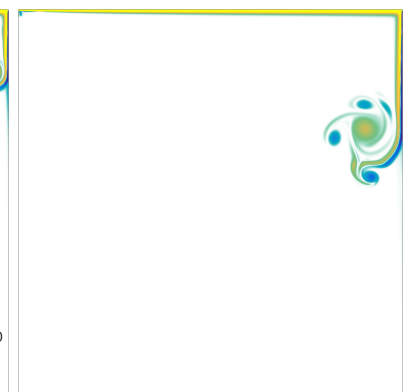

(b) $t=5$; grid: $512^{2}$

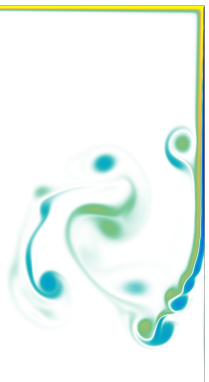

(c) $t=15$; grid: $512^{2}$

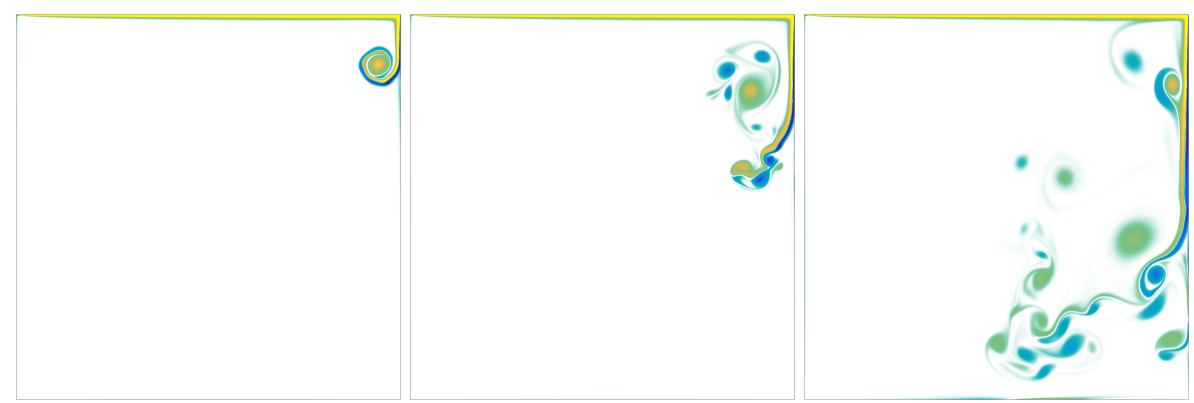

(d) $t=2$; grid: $1024^{2}$

(e) $t=5$; grid: $1024^{2}$

(f) $t=15$; grid: $1024^{2}$

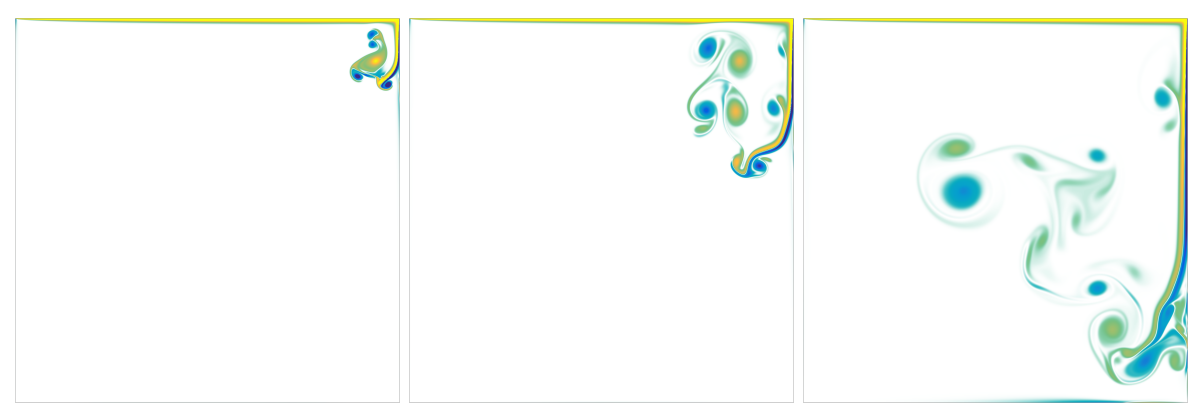
(g) $t=2$; grid: $2048^{2}$
(h) $t=5$; grid: $2048^{2}$
(i) $t=15$; grid: $2048^{2}$

Fig. 32 -D lid-driven flow. Evolution of the vorticity field $-50<\omega_{z}<-50$ for Reynolds number $R e=100000$ at times $t=2, t=5, t=15$ (from left to right). The artificial sound speed $c=3\|\boldsymbol{u}\|_{\infty}^{2}$.

most unstable wavelength [27]. $\|\boldsymbol{u}\|_{\infty}=1$ and $c_{\text {noise }}=10^{-3}$ are taken. With the above parameters, the Reynolds number of the flow can be evaluated as

$$
R e=\frac{\|\boldsymbol{u}\|_{\infty} \delta_{0}}{\nu}=10000 .
$$

The bilinear quadrilateral elements are employed for the simulation in a sequence of meshes $40^{2}, 80^{2}, 160^{2}, 240^{2}, 320^{2}$. The mesh refinement study is carried for the artificial sound speed $c=5\|\boldsymbol{u}\|_{\infty}^{2}$.

In [20] it is reported that the Pressure Stabilised Petrov-Galerkin (PSPG) method fails to converge at the finest grid of $240 \times 240$ elements, whilst in the 


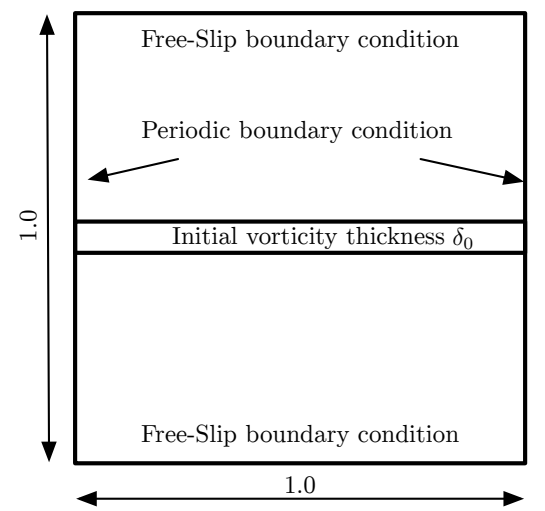

Fig. 4 Schematic show of the plane mixing layer problem

Table 1 Computational settings for 2D plane mixing layer, running on a Desktop machine

\begin{tabular}{lllll}
\hline Elements & Time step $\Delta t$ & ASS & CPU time (hrs) & Peak memory usage (MB) \\
\hline $40^{2}$ & $3.2724 \times 10^{-3}$ & $5\|\boldsymbol{u}\|_{\infty}$ & 0.0066 & 0.5 \\
$80^{2}$ & $1.6362 \times 10^{-3}$ & $5\|\boldsymbol{u}\|_{\infty}$ & 0.053 & 1.9 \\
$160^{2}$ & $4.9087 \times 10^{-4}$ & $5\|\boldsymbol{u}\|_{\infty}$ & 0.43 & 7.8 \\
$240^{2}$ & $1.6362 \times 10^{-4}$ & $5\|\boldsymbol{u}\|_{\infty}$ & 1.44 & 17.5 \\
$320^{2}$ & $9.66 \times 10^{-4}$ & $5\|\boldsymbol{u}\|_{\infty}$ & 3.45 & 31.1 \\
$320^{2}$ & $3.40 \times 10^{-3}$ & $2.5\|\boldsymbol{u}\|_{\infty}$ & 1.09 & 31.1 \\
$320^{2}$ & $2.77 \times 10^{-4}$ & $10\|\boldsymbol{u}\|_{\infty}$ & 13.4 & 31.1 \\
$320^{2}$ & $7.97 \times 10^{-5}$ & $20\|\boldsymbol{u}\|_{\infty}$ & 46.5 & 31.1 \\
\hline
\end{tabular}

proposed method the solution is stable for all meshes. Four different stages can be distinguished, which can be seen in Fig. 5. Four Kelvin-Helmholtz vortices will appear along the $x$-axis, that merge into two vortices and then become one.

1. Development of four primary eddies. The four primary eddies can be seen at time unit 16. This development starts similarly for group $80^{2}, 160^{2}, 240^{2}, 320^{2}$. For the coarsest mesh $40^{2}$, these stages cannot be observed.

2. Pairing of four primary eddies. It can be seen that, after the mesh refinement, the pairing starts later. At time unit 43, the different developments of both pairings can be observed.

3. Pairing of two secondary eddies. The second pairing is finished at about 80 time units, which is close to the results in [25] (75 time units), and earlier in comparison to [20] (115 time units) and [24] (140 time units)

4. Rotation of the final eddy. At the end of the second pairing, the final eddy rotates at a rather fixed position. The value of the vorticity thickness oscillates during this stage due to the elliptic shape of this vortex.

The integrated kinetic energy is given by

$$
E_{k}=\frac{1}{|\Omega|} \int_{\Omega} \frac{\boldsymbol{u} \cdot \boldsymbol{u}}{2} d \Omega
$$

for comparison purposes. The temporal evolution of the kinetic energy dissipation rate is defined as

$$
\epsilon=-\frac{d E_{k}}{d t}
$$




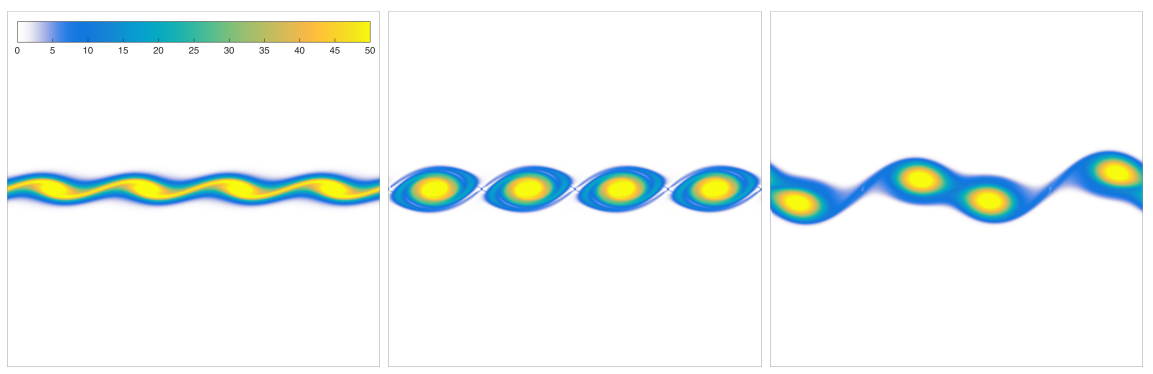

(a) 10

(b) 20

(c) 40

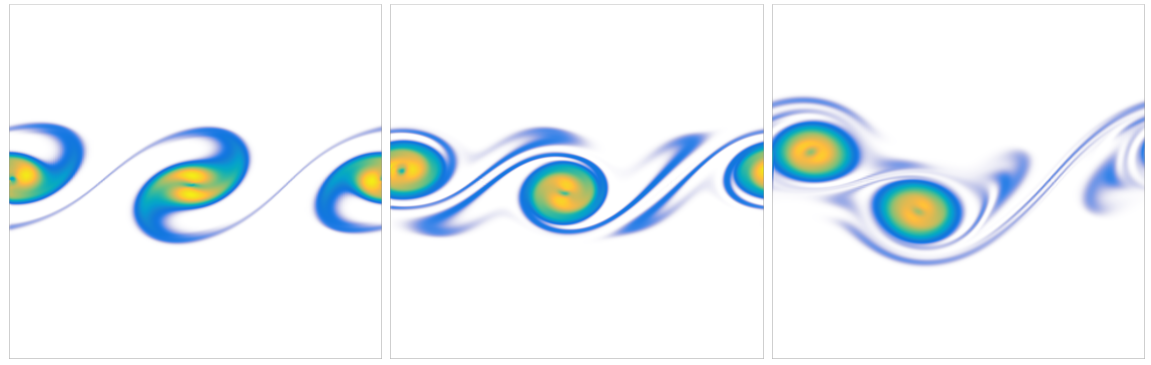

(d) 50

(e) 60

(f) 70

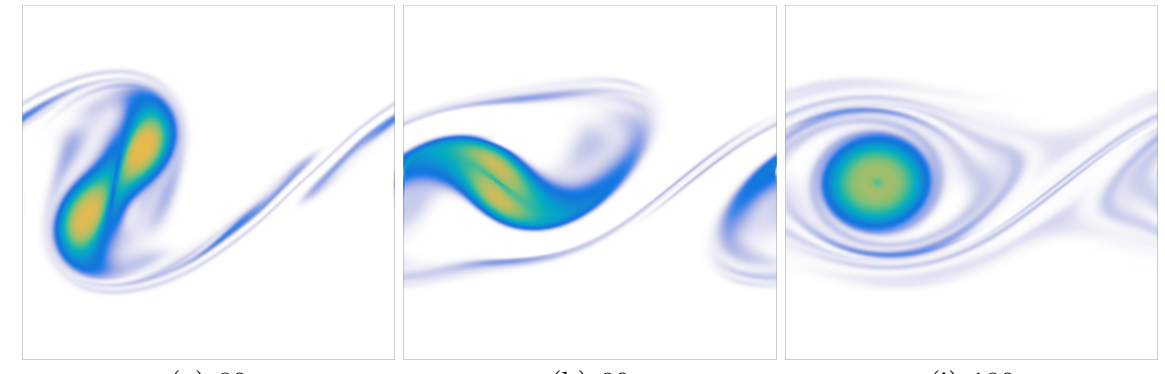

(g) 80

(h) 90

(i) 180

Fig. 52 D plane mixing layer. Coloured vorticity $0<\omega<50$ of the velocity field for the finest mesh grid at time unit: $10,20,40,50,60,70,80,90,180$ in the mesh $320^{2}$. The artificial sound speed $c=5\|\boldsymbol{u}\|_{\infty}^{2}$.

The vorticity thickness is also evaluated quantitatively. The scalar vorticity is defined as

$$
\omega(x, y, t)=\frac{1}{2}\left(\frac{\partial u_{y}}{\partial x}-\frac{\partial u_{x}}{\partial y}\right)
$$

Then, define the maximum vorticity $\omega_{\max }$ as

$$
\omega_{\max }(t)=\sup _{y \in[0,1]} \int_{0}^{1} \omega(x, y, t) d x
$$


where $\int_{0}^{1} \omega(x, y, t) d x$ denotes the integral mean in the periodic $x-$ direction. The vorticity thickness $\delta(t)$ is defined as

$$
\delta(t)=\frac{2 U_{\max }}{\omega_{\max }(t)} .
$$

\subsubsection{Mesh refinement}

Fig. 6 shows the time history of the kinetic energy, the kinetic energy dissipation rate and the vorticity thickness under mesh refinement. The eddies pairing causes the increases of the vorticity thickness, as well as the energy dissipation. For the coarsest mesh size $40 \times 40$, the simulation cannot capture the four and two eddies periods and it can only capture the final large eddy. The mesh of $80 \times 80$ elements is sufficient to clearly observe the development of four stages. The pairing happens relatively slower after refining the mesh.

\subsubsection{Effect of artificial sound speed}

The effect of the different artificial sound speed under meshsize $320 \times 320$ is shown in Fig. 7. It can be seen that ASS $=5$ is enough to capture the key features of the problem. Using the lower ASS number, higher kinetic energy dissipation rate and oscillation can be observed.

\subsection{Three-dimensional laminar flow: lid-driven cavity}

The 3D lid-driven cavity flow illustrated in Fig. 8 is studied to examine the performance of the proposed method and the implementation. The domain in physical space is $\Omega=[0,1] \times[0,1] \times[0,1]$. The top wall moves at a velocity $u_{x}=1, u_{y}=0, u_{z}=0$. The bottom and four vertical walls are non-slip boundaries. A $100^{3}$ uniform structured mesh for $R e=100$ and $R e=1000$ is employed for the computation of the laminar flow. Fig. 9 shows the isosurfaces of magnitude of the velocity $|\boldsymbol{u}|=0.15$ when the flow is reached to the steady state. The artificial sound speed is chosen to be $c=2\|\boldsymbol{u}\|_{\infty}^{2}$, as in the 2D. Quantitive velocity profiles were compared with the reference by Yang [32].

\subsubsection{One-dimensional profile}

The velocity profiles on the mid-plane $y=0.5$ centrelines is illustrated in Fig. 10 for the quantitive comparisons. A good agreement has been reached with the results obtained by Yang [32].

\subsubsection{Two-dimensional profile}

The velocity magnitude in the mid-plane $y=0.5$ and its corresponding pressure field is shown in Fig. 11. The pressure field is stable using the equal velocity and pressure interpolation, which qualitatively agrees well with the results of [18].

In order to show the flow patterns of the cubic cavity flow, streamlines on the plane of $x=0.5, y=0.5$ and $z=0.5$ are presented in Fig. 12. The effects of 


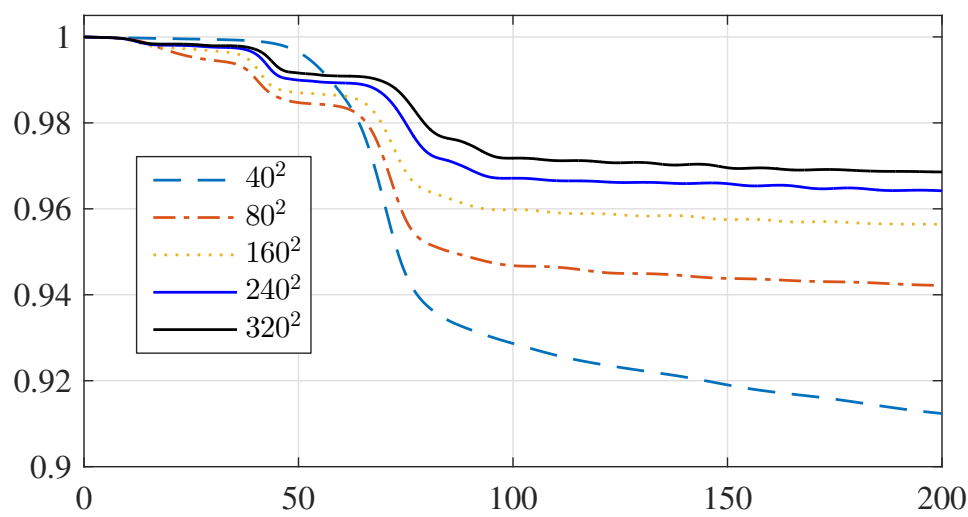

(a) Evolution of the total kinetic energy

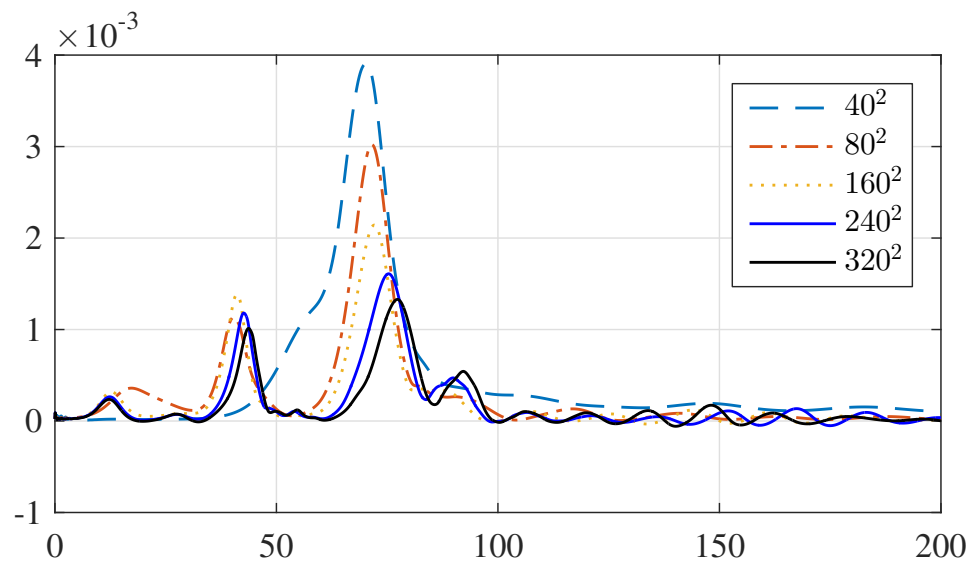

(b) Evolution of the kinetic energy dissipation rate

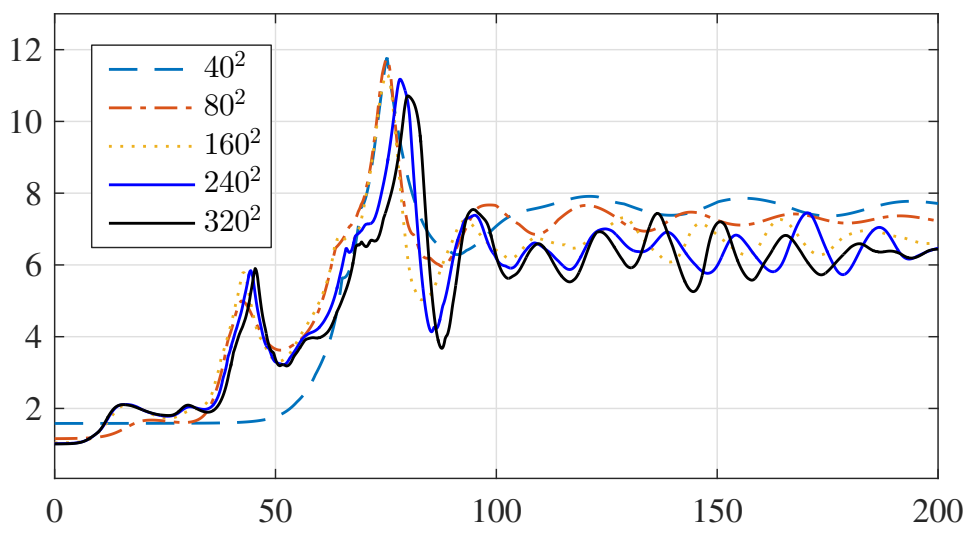

(c) Evolution of the vorticity thickness

Fig. 6 2D plane mixing layer. Time history of the kinetic energy, the kinetic energy dissipation rate and the vorticity thickness for a set of Q1Q1 elements $40^{2}, 80^{2}, 160^{2}, 240^{2}, 320^{2}$, the artificial sound speed $c=5\|\boldsymbol{u}\|_{\infty}^{2}$ 


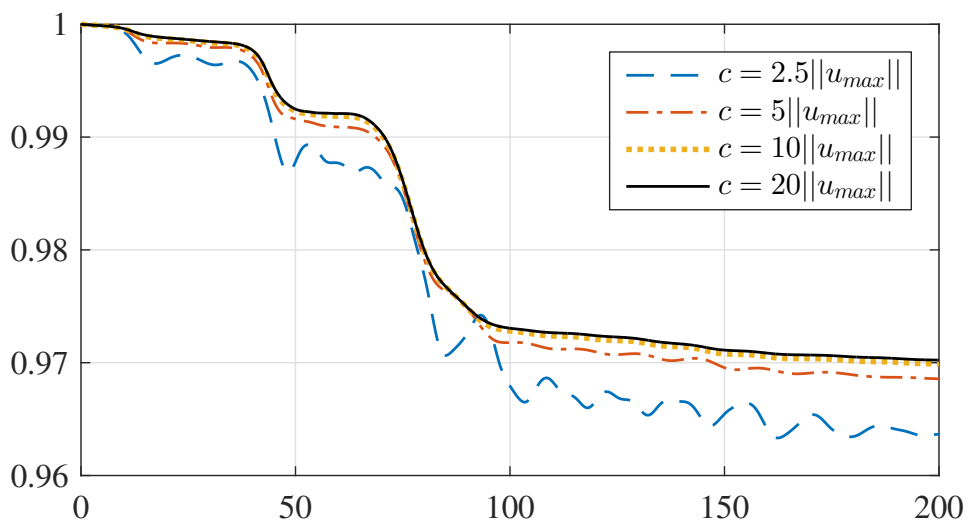

(a) Evolution of the total kinetic energy

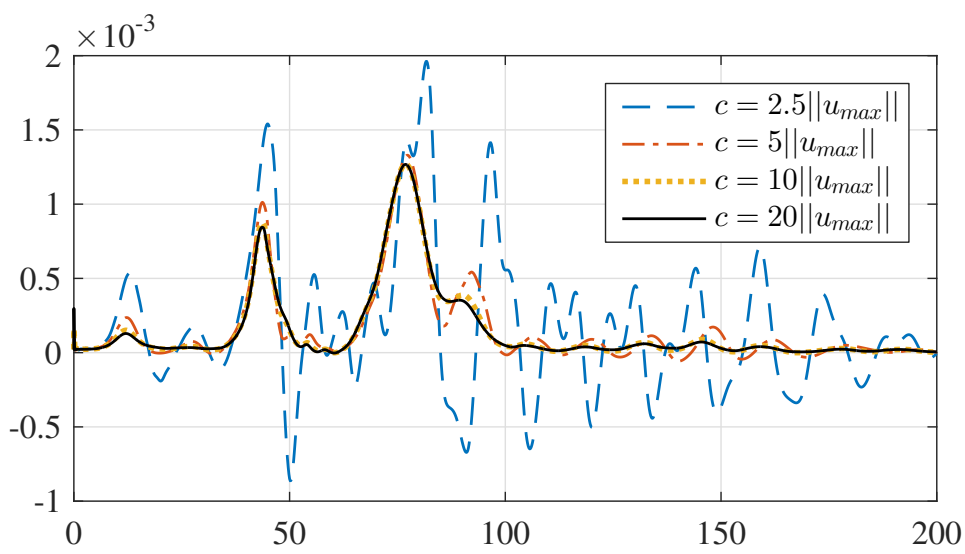

(b) Evolution of the kinetic energy dissipation rate

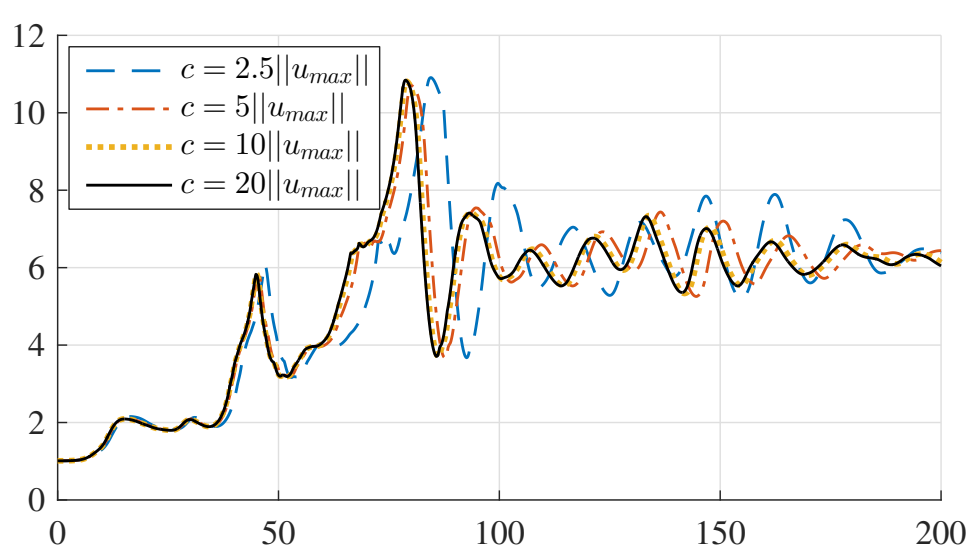

(c) Evolution of the vorticity thickness

Fig. 72 D plane mixing layer. The effect of different artificial speed $c=2.5\|\boldsymbol{u}\|_{\infty}^{2}, c=5\|\boldsymbol{u}\|_{\infty}^{2}$, $c=10\|\boldsymbol{u}\|_{\infty}^{2} c=20\|\boldsymbol{u}\|_{\infty}^{2}$ for meshsize $320^{2}$. 

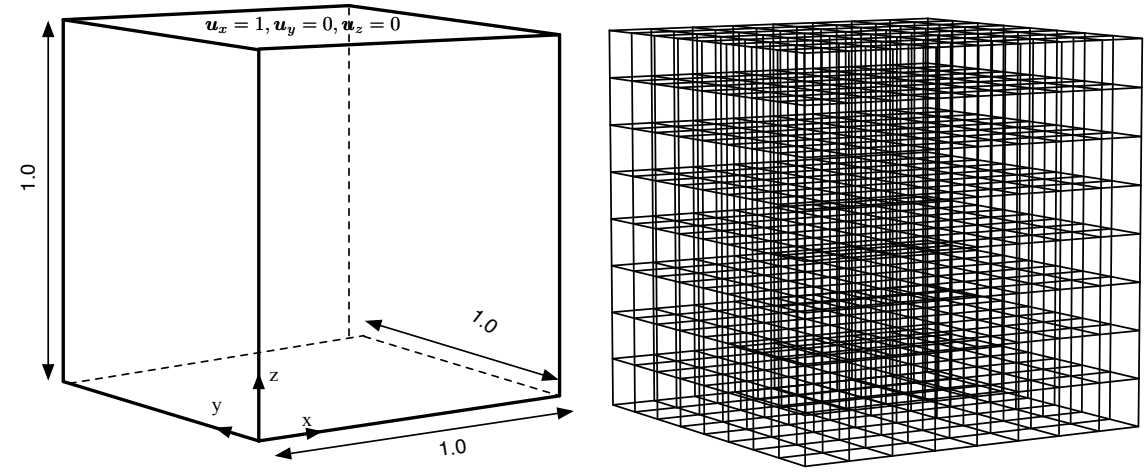

Fig. 8 Schematic show of the 3D lid-driven cavity flow

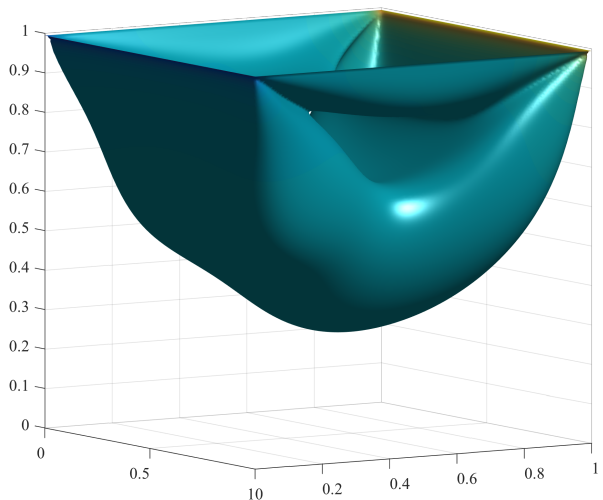

(a) $R e=100$

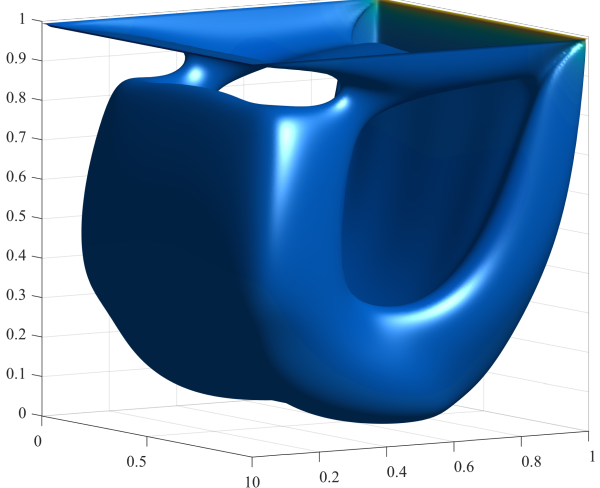

(b) $R e=1000$

Fig. 93 lid-driven cavity. Isosurfaces of magnitude of velocity $|\boldsymbol{u}|=0.15$, with $100^{3}$ trilinear Q1Q1 elements for Reynolds numbers $R e=100$ and $R e=1000$.

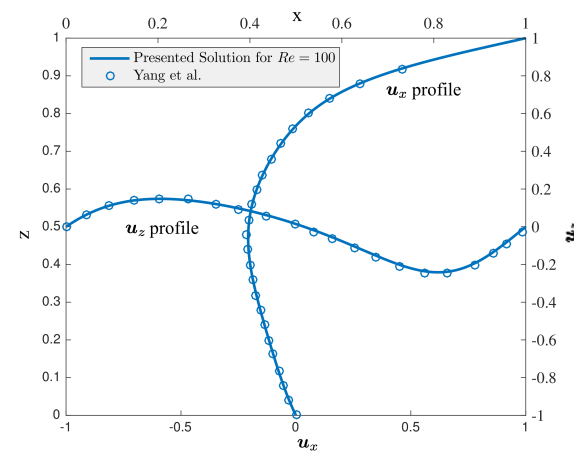

(a) $R e=100$

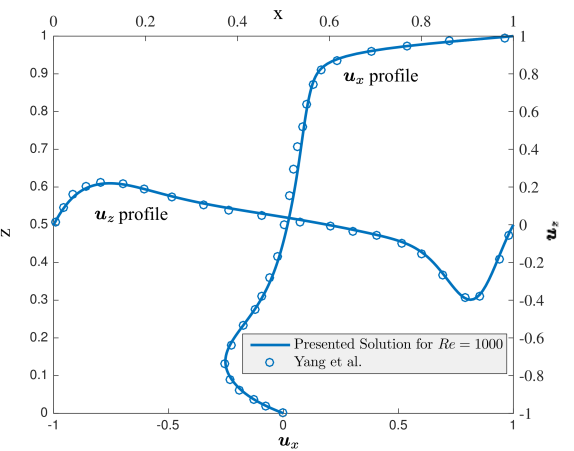

(b) $R e=1000$

Fig. 10 3D lid-driven cavity. Comparison of velocity profiles on the mid-plane $y=0.5$ with reference data [32] for Reynolds number of 100 and 1000. The artificial sound speed $c=2\|\boldsymbol{u}\|_{\infty}^{2}$. 


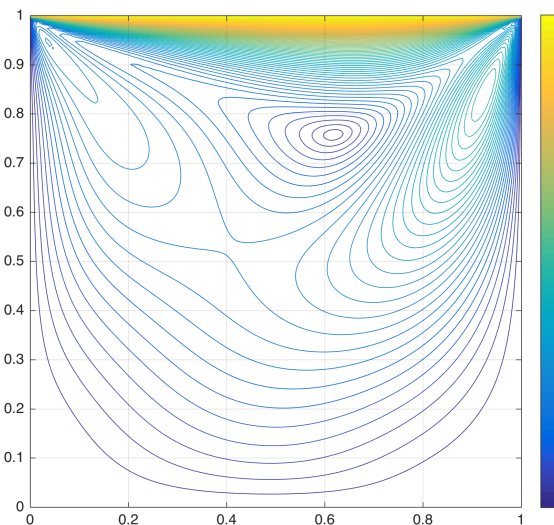

(a) Velocity magnitude, $R e=100$

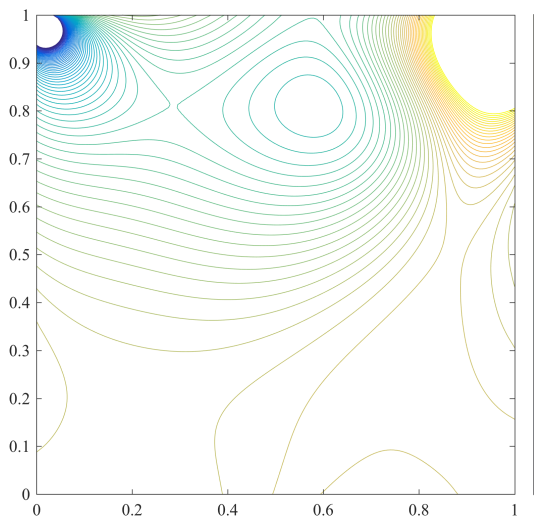

(c) Pressure field, $R e=100$

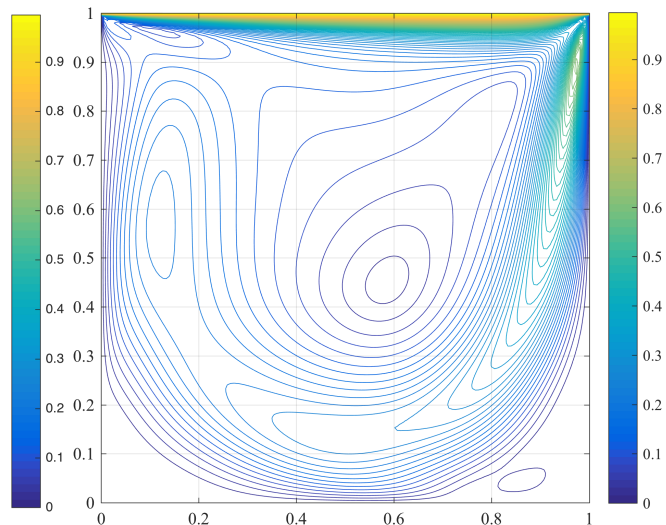

(b) Velocity magnitude, $R e=1000$

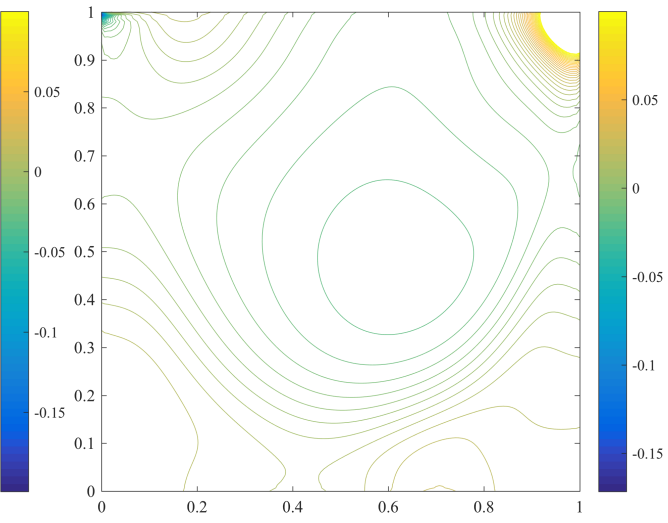

(d) Pressure field, $R e=1000$

Fig. 11 3D lid-driven cavity. Isolines of velocity magnitude and pressure field on the mid-plane $y=0.5$ of cubic cavity at $R e=100$ and $R e=1000$. The artificial sound speed $c=2\|\boldsymbol{u}\|_{\infty}^{2}$.

Reynolds numbers on the flow patterns can be observed. As the Reynolds number increases, the strength of the secondary vortices is enhanced. A similar flow patten was reported in [18]. 


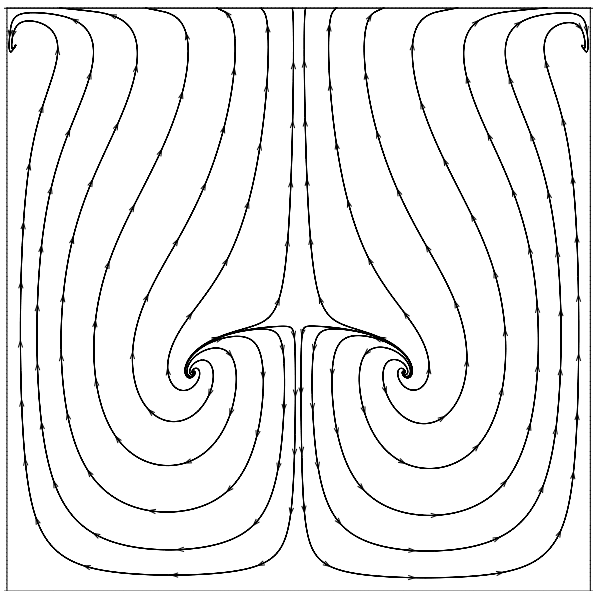

(a) $R e=100$ on the mid-plane $x=0.5$

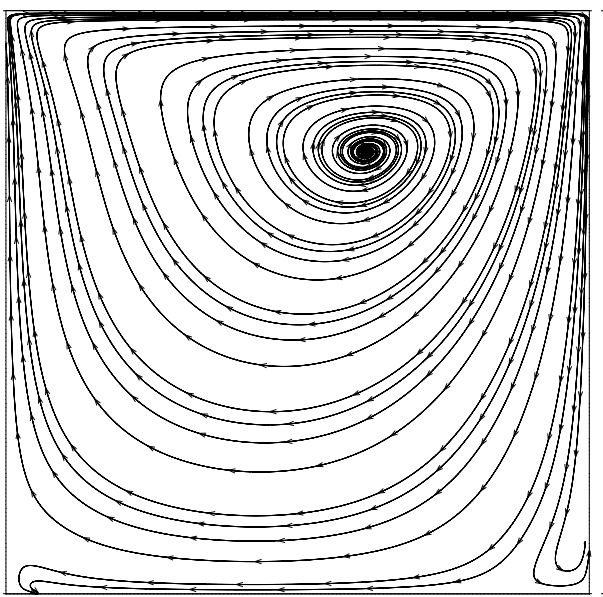

(c) $R e=100$ on the mid-plane $y=0.5$

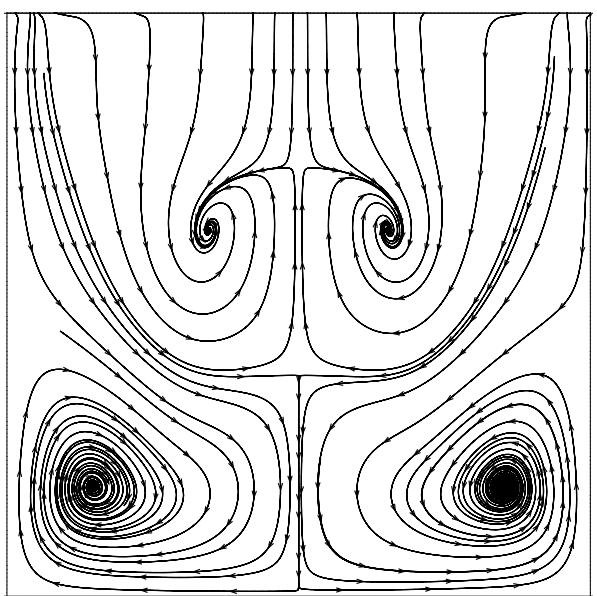

(b) $R e=1000$ on the mid-plane $x=0.5$

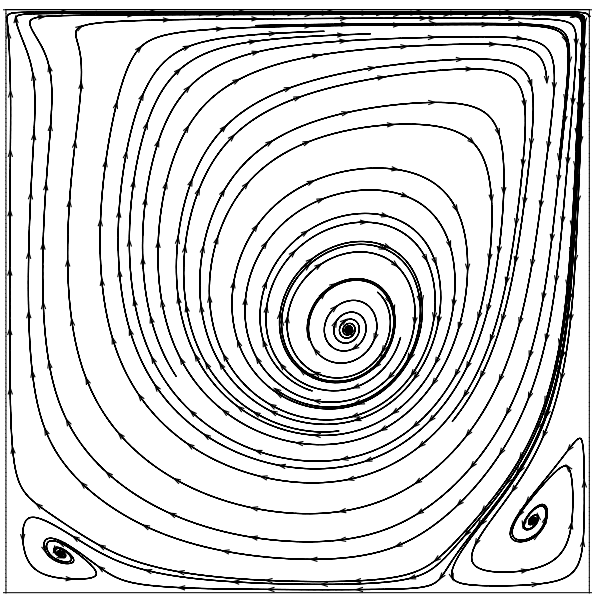

(d) $R e=1000$ on the mid-plane $y=0.5$

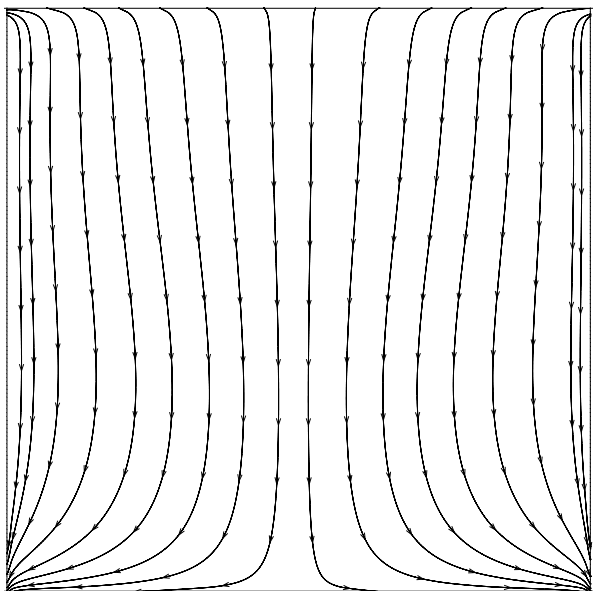

(e) $R e=100$ on the mid-plane $z=0.5$

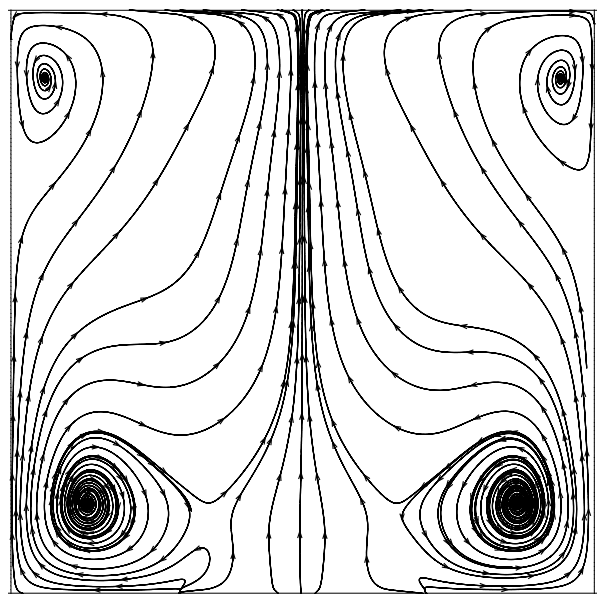

(f) $R e=1000$ on the mid-plane $z=0.5$

Fig. 12 3D lid-driven cavity. Streamlines on the mid-plane $x=0.5, y=0.5$ and $z=0.5$ of the cubic cavity at Reynolds number of 100 and 1000. The artificial sound speed $c=2\|\boldsymbol{u}\|_{\infty}^{2}$. 
4.4 Three-dimensional turbulent flow: Taylor-Green vortex

The Taylor-Green vortex problem is widely used in the numerical simulation of turbulent flow. This flow transits to turbulence and represents a basic turbulence decay mode similar to decaying homogeneous turbulence $[8,7]$. The domain in physical space is $\Omega=[0,2 \pi] \times[0,2 \pi] \times[0,2 \pi]$ with periodic boundary conditions in all directions. Within the domain, initial velocity and pressure is defined as

$$
\begin{aligned}
u_{x} & =u_{0} \cos (x) \sin (y) \sin (z) \\
u_{y} & =-u_{0} \sin (x) \cos (y) \sin (z) \\
u_{z} & =0 \\
p & =p_{0}+\frac{1}{16}(\sin (\cos (2 x)+\cos (2 y))(\cos (2 z)+2)
\end{aligned}
$$

where $u_{0}=1$. The initial velocity field on the Fourier space has eight modes located at the wave number $\boldsymbol{k}=( \pm 1, \pm 1, \pm 1)$. The Reynolds number of flow is defined as $R e=\frac{u_{0} L}{\nu}$ and is equal to 1600 .

The primary method for evaluating the TGV solution is examining the energy dissipation rate. The integrated kinetic energy is given by

$$
E_{k}=\frac{1}{|\Omega|} \int_{\Omega} \frac{\boldsymbol{u} \cdot \boldsymbol{u}}{2} d \Omega
$$

The temporal evolution of the kinetic energy dissipation rate is defined as

$$
\epsilon_{1}=-\frac{d E_{k}}{d t}
$$

A second kinetic energy dissipation rate can be defined from the integrated enstrophy of the problem:

$$
\epsilon_{2}=\frac{2 \nu}{|\Omega|} \int_{\Omega} \frac{\boldsymbol{\omega} \cdot \boldsymbol{\omega}}{2} d \Omega
$$

where $\boldsymbol{\omega}$ is the vorticity of the velocity field.

Trilinear elements are employed for the simulation in a sequence of meshes $100 \cdot 2^{i} \times 128 \cdot 2^{i}$ for $i=1,2,3$. All the simulation were run on a single processor desktop, including the case for $512^{3}$. The details of each cases setting were given in Table 2. We also report the peak memory consumption for every run. In Fig. 13 we present some vorticity iso-surface images. The initial condition Fig. 13(a) has eight vortices with the same scale corresponding to the eight Fourier modes. The vorticity iso-surfaces are very similar to the results obtained by the implicit VMS [16].

\subsubsection{Effect of artificial sound speed}

The effect of the artificial sound speed (ASS) was tested under a mesh size of $100^{3}$. The ASS is chosen to be 3,5,10,20 times the maximum of the velocity $\|\boldsymbol{u}\|_{\infty}$. Fig. 14 shows the effect of ASS on the resolved energy, the total kinetic energy evolution and dissipation rate. It can be observed from that the resolved dissipation is very similar for all the cases. However, the energy oscillation can be seen from time $t=0$ to $t=3$ due to the artificial compressibility scheme. As the ASS number increases, 


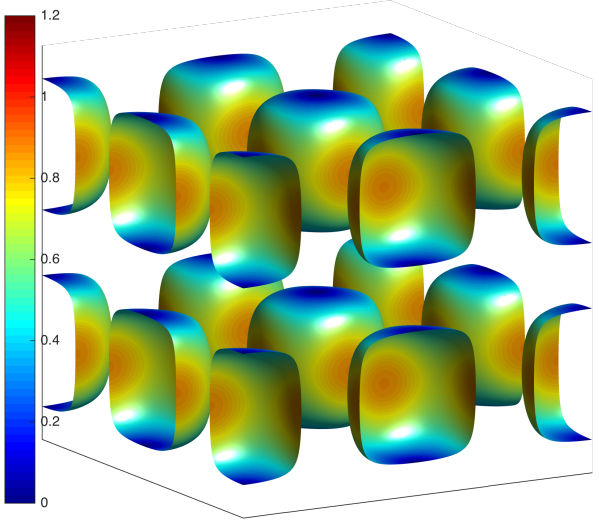

(a) Isosurface for $|\boldsymbol{\omega}|=1$ at $t=0.0$

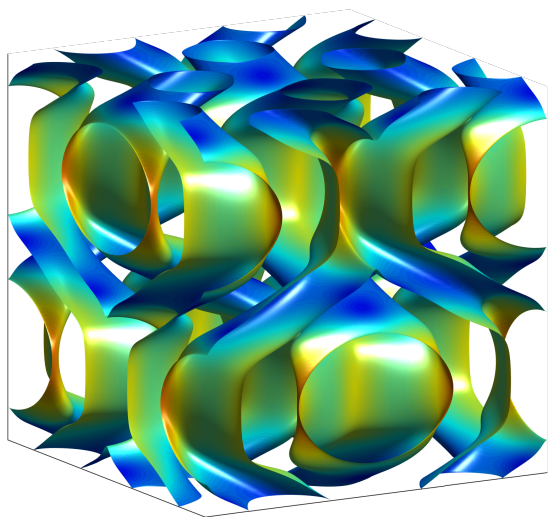

(b) Isosurface for $|\boldsymbol{\omega}|=1$ at $t=2.0$

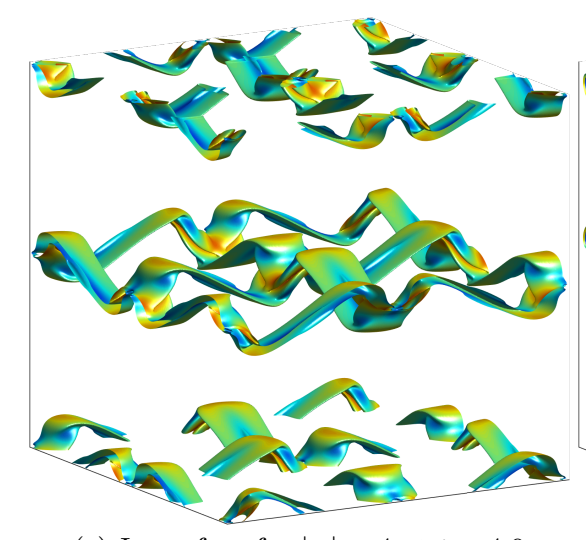

(c) Isosurface for $|\boldsymbol{\omega}|=4$ at $t=4.0$

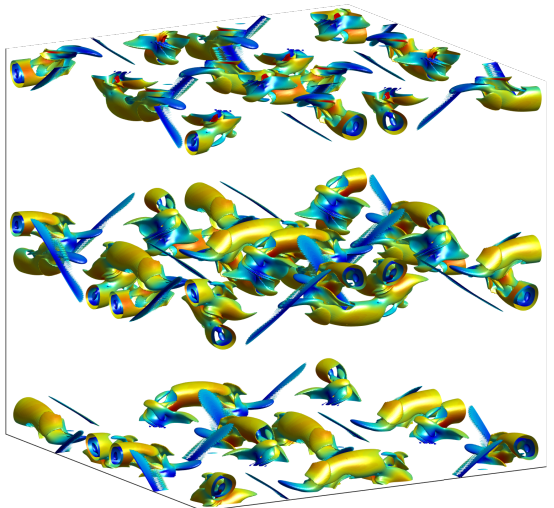

(d) Isosurface for $|\boldsymbol{\omega}|=7$ at $t=6.0$

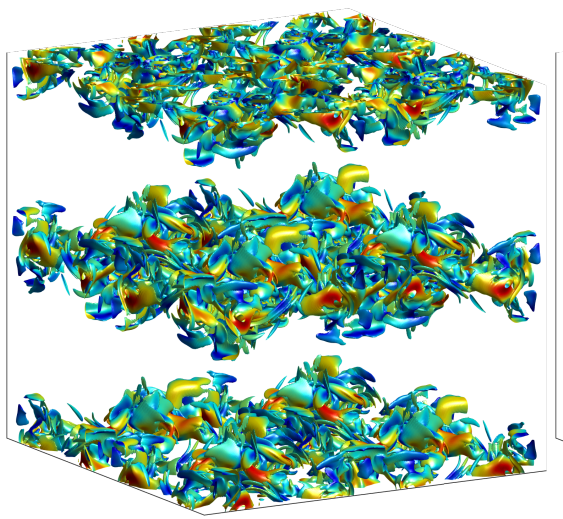

(e) Isosurface for $|\boldsymbol{\omega}|=8$ at $t=9.0$

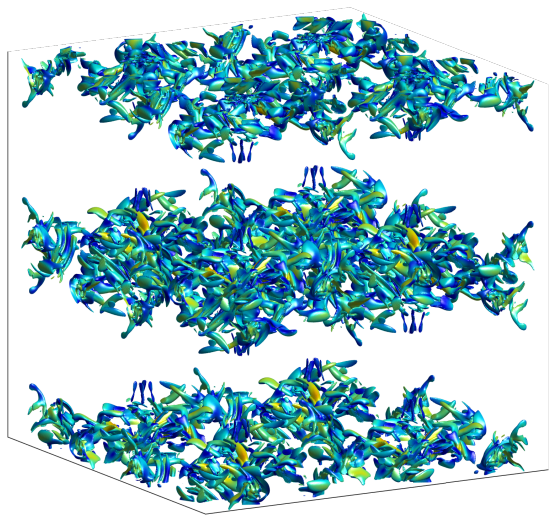

(f) Isosurface for $|\boldsymbol{\omega}|=9$ at $t=11.0$

Fig. 13 Taylor-Green vortex. Vorticity isosurfaces with velocity colored at different time step, mesh of $512^{3}$ trilinear elements, the artificial sound speed $c=5\|\boldsymbol{u}\|_{\infty}^{2}$. 
Table 2 Computational settings for 3D Taylor Green vortex, run on a 12 cores machines, speed up by OpenMP

\begin{tabular}{lllll}
\hline Elements & Time step $\Delta t$ & ASS & CPU time (hrs) & Peak memory usage (GB) \\
\hline $128^{3}$ & $1.63 \times 10^{-3}$ & $3\|\boldsymbol{u}\|_{\infty}$ & 1 & 0.68 \\
$128^{3}$ & $4.90 \times 10^{-4}$ & $5\|\boldsymbol{u}\|_{\infty}$ & 3 & 0.68 \\
$128^{3}$ & $1.64 \times 10^{-4}$ & $10\|\boldsymbol{u}\|_{\infty}$ & 11 & 0.68 \\
$128^{3}$ & $3.08 \times 10^{-5}$ & $20\|\boldsymbol{u}\|_{\infty}$ & 55 & 0.68 \\
$256^{3}$ & $2.46 \times 10^{-4}$ & $5\|\boldsymbol{u}\|_{\infty}$ & 16 & 5.44 \\
$512^{3}$ & $1.23 \times 10^{-4}$ & $5\|\boldsymbol{u}\|_{\infty}$ & 253 & 43.51 \\
\hline
\end{tabular}

the oscillation decreases, as the relaxation of the incompressibility becomes strict. As expected, the large ASS number brings the time stepping restrictions and causes more computational time. Table 2 also illustrates the computation times required for the different ASS values. For the Taylor-Green vortex problem, the interest is to study the transition period from the laminar to turbulent flow and the turbulence decay. The energy oscillation caused by the artificial compressibility scheme is very small before the turbulent transition. As a result, ASS equal to $5\|\boldsymbol{u}\|_{\infty}$ is chosen for the mesh refinement simulations.

\subsubsection{Mesh refinement}

Fig. 15 shows the total kinetic energy and the total energy dissipation rate. The directly computing energy dissipation rate is well predicated compared with the DNS result by $[17,7]$. This indicates that the turbulent structures are under-resolved and the sub-grid scales plays a significant role in the energy dissipation. And the proposed explicit variational multiscale method is able to approximate the un-resolved scales and simulate the turbulent flow.

\subsubsection{Energy spectra}

The progression of the energy spectra with time on $512^{3}$ is shown in Fig. 16(a). At $t=2$ all the energy is confined to small wave numbers. As the time progresses, the energy begins to cascade down to smaller scales. The energy in the smallest scales peaks around $t=10$. This corresponds to the maximum energy dissipation rate. The spectra for $t=10$ for a set of meshes are shown in Fig. 16(b). The finer grid is able to capture smaller energy scales.

\section{Conclusions}

In this work, we have proposed a pseudo-compressible VMS solver for turbulent incompressible flows. As a result, VMS-type LES models can now be combined with explicit time stepping techniques. We have analyzed the resulting scheme for a set of benchmark tests. Out of these results, we can conclude that one can obtain accurate under-resolved LES simulations of turbulent flows by using the proposed explicit VMS scheme with a very limited memory consumption. In particular, the transient numerical examples, $2 D$ Kelvin-Helmholtz instability and $3 D$ Taylor-Green Vortex, show a good convergence with respect to the artificial compressibility sound speed and the mesh size. 


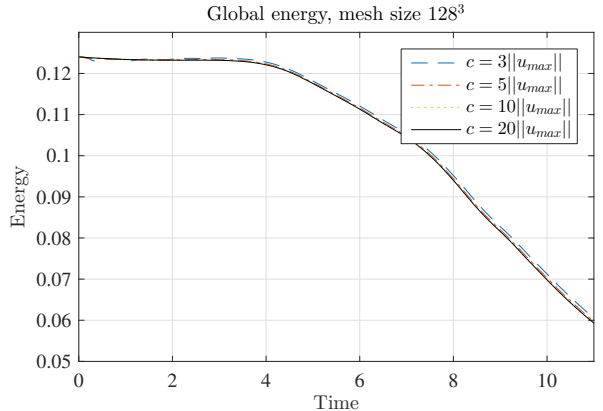

(a) Total kinetic energy

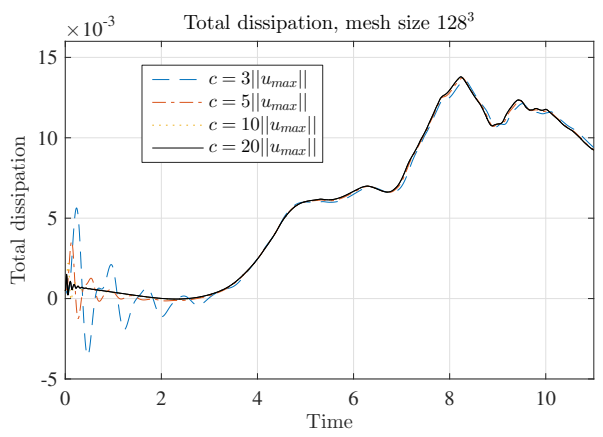

(c) Total kinetic dissipation rate

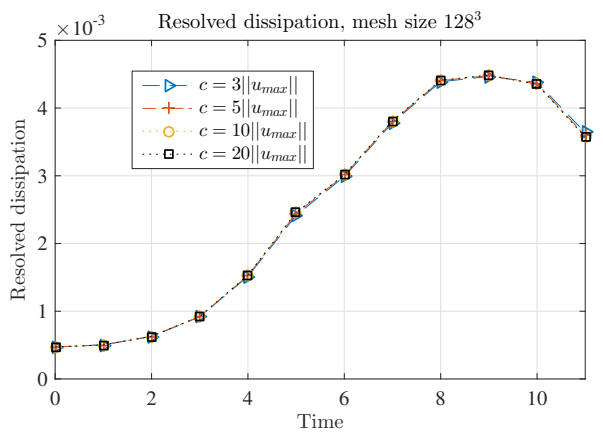

(e) Resolved dissipation rate

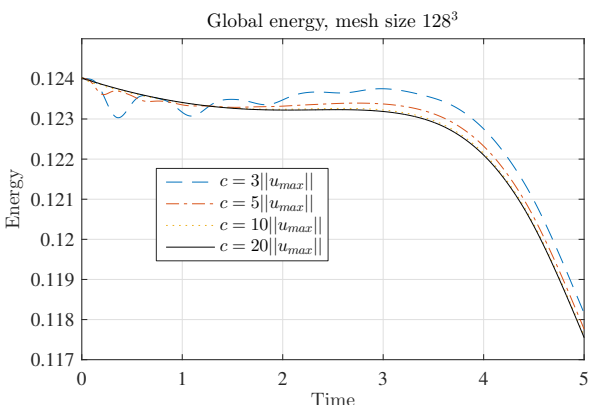

(b) Close view

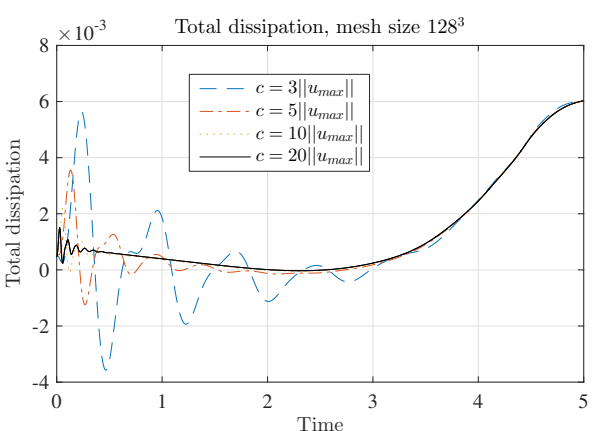

(d) Close view

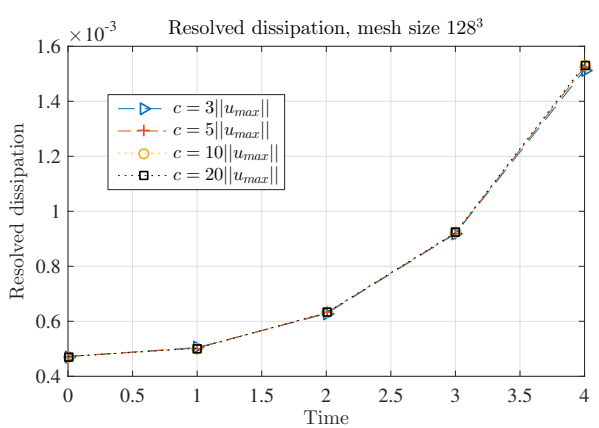

(f) Close view

Fig. 14 Taylor-Green vortex. Study the effect of different artificial sound speed for resolved energy, total kinetic energy evolution and dissipation rate under grid $128^{3}$.

Acknowledgements S. Badia's work has been partially funded by the European Research Council under the FP7 Program Ideas through the Starting Grant No. 258443 - COMFUS: Computational Methods for Fusion Technology and the FP7 NUMEXAS project under grant agreement 611636 . S. Badia and R. Codina gratefully acknowledge the support received from the Catalan Government through the ICREA Acadèmia Research Program. 


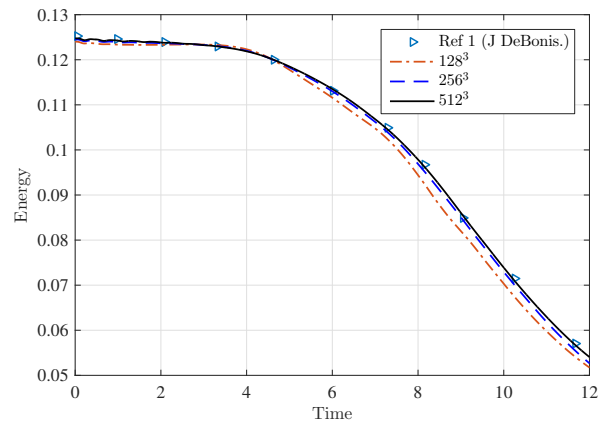

(a) Total kinetic energy

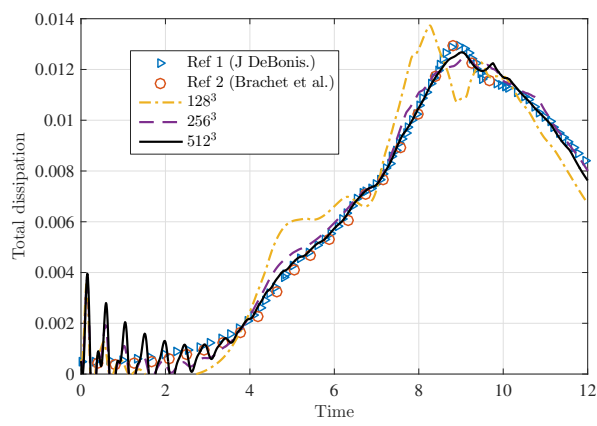

(b) Total kinetic dissipation rate

Fig. 15 Taylor-Green vortex. The evolution of the total kinetic energy and the kinetic dissipation rate under mesh refinement, the artificial sound speed $c=5\|\boldsymbol{u}\|_{\infty}^{2}$.

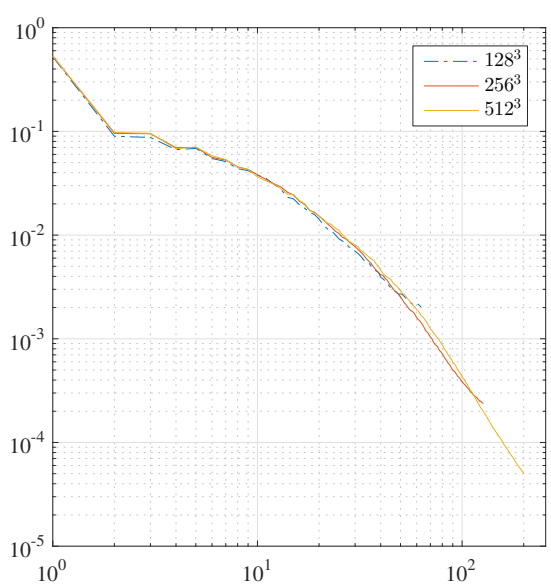

(a) Energy spectrum under different mesh size at $t=10$

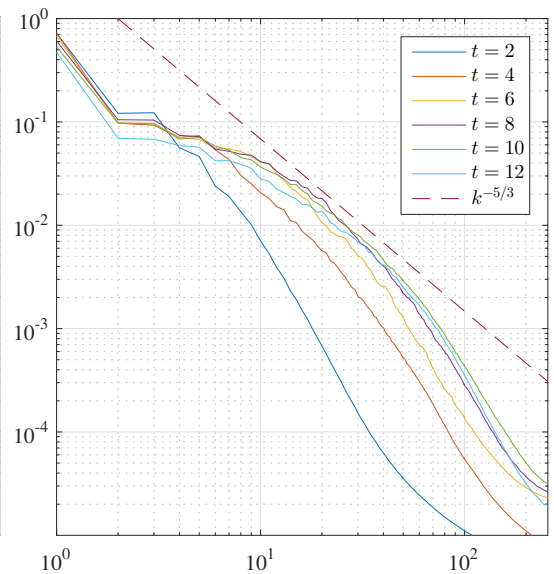

b) Evolution of the energy spectra under different time on the $512^{3}$ grid

Fig. 16 Taylor-Green vortex. Energy spectrum for the Taylor-Green Vortex problem, the artificial sound speed $c=5\|\boldsymbol{u}\|_{\infty}^{2}$.

\section{Appendix}

Here we illustrate the matrix-vector implementation in 2D for linear FEs for simplicity. The nodal shape function associated to node $a$ is denoted by $N^{a}(x)$ and its value at gauss point $b$ is represent as $N_{b}^{a}$. Every node $a$ is represented by the lexicographical index $\left(i_{a}, j_{a}\right)$, with $i_{a}, j_{a}=0,1$. Further, given the $\alpha$-th axis direction, with $\alpha=1,2$, we define the function $t w(a, \alpha)=t w\left(i_{a}, j_{a}, \alpha\right)$ as $\left(1-i_{a}, j_{a}\right)$ for $\alpha=1$ and $\left(i_{a}, 1-j_{a}\right)$ for $\alpha=2$. We use a nodal quadrature for the numerical integration, which leads to a lumped mass matrix. As a result, using the expression 
of the nodal shape functions and their derivatives on nodes, we get:

$$
\begin{aligned}
& N^{a}\left(x_{b}\right)=\delta_{a b}, \quad N^{a}\left(x_{b}\right)=\delta_{a b} \quad \partial_{\alpha} N^{a}\left(x_{b}\right)=-\partial_{\alpha} N^{t w(a, \alpha)}\left(x_{b}\right), \\
& \partial_{\alpha} N^{a}\left(x_{t w(a, \beta)}\right)=\delta_{\alpha \beta} \partial_{\alpha} N^{a}\left(x_{a}\right),
\end{aligned}
$$

where $x_{b}$ denotes the coordinates of the node $b$ and $\delta_{\alpha \beta}$ the Kronecker delta. Using these expressions, we can implement efficiently all the terms in our matrixfree formulation using the following expressions. The viscous term is implemented as follows:

$$
\begin{aligned}
& \int_{K} \partial_{\beta} u^{\alpha}(x) \partial_{\beta} N^{a}(x) \mathrm{d} \Omega=\sum_{b=1}^{4} \partial_{\beta} u^{\alpha}\left(x_{b}\right) \partial_{\beta} N_{b}^{a}|J| w_{b} \\
& =\left(\partial_{\beta} u^{\alpha}\left(x_{a}\right)+\partial_{\beta} u^{\alpha}\left(x_{t w(a, \beta)}\right)\right) \partial_{\beta} N_{a}^{a}|J| w_{a} .
\end{aligned}
$$

The nonlinear convective term is implemented as:

$$
\begin{aligned}
& \int_{K} a_{\beta} \partial_{\beta} u^{\alpha}(x) a_{\gamma} \partial_{\gamma} N^{a}(x) \mathrm{d} \Omega=\sum_{b=1}^{4}\left(a_{\beta} \partial_{\beta} u^{\alpha}\right)\left(x_{b}\right) a_{\gamma}\left(x_{b}\right) \partial_{\gamma} N_{b}^{a}|J| w_{b} \\
& =\left(\left(a_{\beta} \partial_{\beta} u^{\alpha} a_{\gamma}\right)\left(x_{a}\right)+\left(a_{\beta} \partial_{\beta} u^{\alpha} a_{\gamma}\right)\left(x_{t w(a, \gamma)}\right)\right) \partial_{\gamma} N_{a}^{a}|J| w_{a} .
\end{aligned}
$$

We proceed analogously for the rest of terms.

\section{References}

1. S. Badia and R. Codina. Algebraic pressure segregation methods for the incompressible Navier-Stokes equations. Archives of Computational Methods in Engineering, 15(3):343$369,2008$.

2. Santiago Badia and Ramon Codina. On a multiscale approach to the transient Stokes problem: Dynamic subscales and anisotropic spacetime discretization. Applied Mathematics and Computation, 207(2):415-433, January 2009.

3. Santiago Badia, Alberto F. Martín, and Javier Principe. A highly scalable parallel implementation of balancing domain decomposition by constraints. SIAM Journal on Scientific Computing, 36(2):C190-C218, January 2014.

4. Santiago Badia, Alberto F. Martín, and Javier Principe. Balancing domain decomposition at extreme scales. SIAM Journal on Scientific Computing, in press, 2015.

5. Y. Bazilevs, V.M. Calo, J.A. Cottrell, T.J.R. Hughes, A. Reali, and G. Scovazzi. Variational multiscale residual-based turbulence modeling for large eddy simulation of incompressible flows. Computer Methods in Applied Mechanics and Engineering, 197(1-4):173201, 2007.

6. B.J. Boersma, M.N. Kooper, T.T.M. Nieuwstadt, and P. Wesseling. Local grid refinement in Large-Eddy Simulations. Journal of Engineering Mathematics, 32(2/3):161-175, 1997.

7. M.E. Brachet. Direct simulation of three-dimensional turbulence in the Taylor-Green vortex. Fluid Dynamics Research, 8(1):1-8, 1991

8. M.E. Brachet, D. Meiron, S. Orszag, B.G. Nickel, R.H. Morf, and U. Frisch. Small-scale structure of the Taylor-Green vortex. Journal of Fluid Mechanics, 130:411-452, 1983.

9. A.J. Chorin. A numerical method for solving incompressible viscous flow problems. Journal of Computational Physics, 2(1):12-26, 1967.

10. R. Codina. Stabilized finite element approximation of transient incompressible flows using orthogonal subscales. Computer Methods in Applied Mechanics and Engineering, 191:4295-4321, 2002.

11. R. Codina and J. Blasco. Analysis of a stabilized finite element approximation of the transient convection-diffusion-reaction equation using orthogonal subscales. Computing and Visualization in Science, 4:167-174, 2002. 
12. R. Codina, J. Principe, and J. Baiges. Subscales on the element boundaries in the variational two-scale finite element method. Computer Methods in Applied Mechanics and Engineering, 198:838-852, 2009.

13. R. Codina, J. Principe, O. Guasch, and S. Badia. Time dependent subscales in the stabilized finite element approximation of incompressible flow problems. Computer Methods in Applied Mechanics and Engineering, 196:2413-2430, 2007.

14. R. Codina and O. Soto. Approximation of the incompressible Navier-Stokes equations using orthogonal subscale stabilization and pressure segregation on anisotropic finite element meshes. Computer Methods in Applied Mechanics and Engineering, 193:1403-1419, 2004.

15. R. Codina and O.C. Zienkiewicz. CBS versus GLS stabilization of the incompressible Navier-Stokes equations and the role of the time step as stabilization parameter. Communications in Numerical Methods in Engineering, 18:99-112, 2002.

16. O. Colomés, S. Badia, R. Codina, and J. Principe. Assessment of variational multiscale models for the large eddy simulation of turbulent incompressible flows. Computer Methods in Applied Mechanics and Engineering, 285:32-63, 2015.

17. James DeBonis. Solutions of the taylor-green vortex problem using high-resolution explicit finite difference methods. AIAA paper, 382:2013, 2013.

18. H Ding, C Shu, KS Yeo, and $\mathrm{D} \mathrm{Xu}$. Numerical computation of three-dimensional incompressible viscous flows in the primitive variable form by local multiquadric differential quadrature method. Computer Methods in Applied Mechanics and Engineering, 195(7):516-533, 2006.

19. U. Ghia, K.N. Ghia, and C.T. Shin. High-Re solutions for incompressible flow using the Navier-Stokes equations and a multigrid method. Journal of Computational Physics, 48(3):387-411, 1982 .

20. V. Gravemeier. The variational mulstiscale method for laminar and turbulent flow. Archives of Computational Mechanics-State of the Art Reviews, 13:249-324, 2006.

21. O. Guasch and R. Codina. Statistical behavior of the orthogonal subgrid scale stabilization terms in the finite element large eddy simulation of turbulent flows. Computer Methods in Applied Mechanics and Engineering, 261-262:154-166, 2013.

22. T.J.R. Hughes, G.R. Feijoo, L. Mazzei, and J.B. Quincy. The variational multiscale method-A paradigm for computational mechanics. Computer Methods in Applied Mechanics and Engineering, 166:3-24, 1998.

23. T.J.R. Hughes, L. Mazzei, and K.E. Jansen. Large eddy simulation and the variational multiscale method. Computing and Visualization in Science, 3:47-59, 2000. $10.1007 / \mathrm{s} 007910050051$.

24. V. John. An assessment of two models for the subgrid scale tensor in the rational LES model. Journal of computational and applied mathematics, 173(1):57-80, 2005.

25. Marcel Lesieur, Chantal Staquet, Pascal Le Roy, and Pierre Comte. The mixing layer and its coherence examined from the point of view of two-dimensional turbulence. Journal of Fluid Mechanics, 192:511-534, 1988.

26. A.G. Malan, R.W. Lewis, and P. Nithiarasu. An improved unsteady, unstructured, artificial compressibility, finite volume scheme for viscous incompressible flows: Part II. Application. International Journal for Numerical Methods in Engineering, 54(5):715-729, 2002.

27. A. Michalke. On the inviscid instability of the hyperbolic tangent velocity profile. Journal of Fluid Mechanics, 19:543-556, 1964.

28. P. Moin and K. Mahesh. Direct numerical simulation: A tool in turbulence research. Annual Review of Fluid Mechanics, 30(1):539-578, 1998.

29. P. Nithiarasu. An efficient artificial compressibility (AC) scheme based on the characteristic based split (CBS) method for incompressible flows. International Journal for Numerical Methods in Engineering, 56(13):1815-1845, 2003.

30. Johann Rudi, A Cristiano I Malossi, Tobin Isaac, Georg Stadler, Michael Gurnis, Peter WJ Staar, Yves Ineichen, Costas Bekas, Alessandro Curioni, and Omar Ghattas. An extreme-scale implicit solver for complex pdes: highly heterogeneous flow in earth's mantle. In Proceedings of the International Conference for High Performance Computing, Networking, Storage and Analysis, page 5. ACM, 2015.

31. D.C. Wilcox. Turbulence Modeling for CFD. Turbulence Modeling for CFD. DCW Industries, Incorporated, 2006.

32. Jaw-Yen Yang, Shih-Chang Yang, Yih-Nan Chen, and Chiang-An Hsu. Implicit weighted eno schemes for the three-dimensional incompressible navier-stokes equations. Journal of Computational Physics, 146(1):464-487, 1998. 